\title{
Arenen für Angriffe oder Arenen der Akzeptanz?
}

\author{
Medien als Akteure in ,Genderismus'-Diskursen
}

DOROTHEE BECK

\section{EINLEITUNG}

„Kretschmann für Gender-Theorie“ titelte die Frankfurter Allgemeine Zeitung am 28.02.2014. ${ }^{1}$ Baden-Württembergs grüner Ministerpräsident hatte sich dafür ausgesprochen, die Akzeptanz geschlechtlicher Vielfalt in die Reform des schulischen Bildungsplans des Landes aufzunehmen. „Papst kritisiert Gender-Lehre an Schulen“ überschrieb Spiegel online am 03.08.2016 einen Artikel, dem zufolge Franziskus gegenüber polnischen Bischöfen bemängelt hatte, Kinder bekämen in der Schule beigebracht, sie könnten sich ihr Geschlecht aussuchen. Der Begriff Gender ist in extrem-rechten, religiös-fundamentalistischen und maskulistischen Kreisen - aber auch in einigen Medien - zum catch-all term für den Kampf gegen die Liberalisierung der Geschlechterordnungen geworden. Insbesondere in seiner Hypostasierung als die Gender-Theorie, die Gender-Ideologie oder die Gender-Lehre imaginiert der Begriff ein einheitliches, absichts- und planvolles Vorgehen der Verfechter_innen ${ }^{2}$ eben dieser Lehre, die, so der Vorwurf, traditionelle, zumeist ,christlich-abendländische“ Werte aus den Angeln heben wollten. Mit der Wortendung, ismus` soll Gender, also die Annahme der sozialen und kulturellen Konstruktion von Geschlecht, als Ideologie gebrandmarkt werden. Der Kampfbegriff ,Genderismus ${ }^{63}$

1 Medien-Artikel, die nicht zum Text-Sample gehören, sowie andere Original-Dokumente aus dem Spektrum der beforschten Akteur_innen sind im Literaturverzeichnis unter „Weitere Primärquellen“ angegeben.

2 In diesem Beitrag nutze ich den Gender-Gap (Unterstrich), um die Vielfalt von Geschlecht auszudrücken.

3 Der Begriff Genderismus wird im Folgenden in einfache Anführungszeichen gesetzt, um die kritische Distanzierung kenntlich zu machen. Nach Scheele (2016: 3) wird der Begriff 
richtet sich gegen Errungenschaften und vermeintliche Verfehlungen von Feminismus und institutionalisierter Gleichstellungspolitik sowie gegen die Gender Studies insgesamt (vgl. Claus 2014; Frey 2014; Gesterkamp 2010; Hark/Villa 2015; Rosenbrock 2012).

Im vorliegenden Beitrag rechne ich jene gesellschaftlichen Akteur_innen, die die Kategorie Gender ablehnen, kurz ,Gender'-Kritiker_innen ${ }^{4}$ genannt, dem Antifeminismus zu. Antifeminismus definiere ich im Anschluss an die Einleitung in diesem Band als Gegenbegriff zu einem herrschaftskritischen, demokratiebezogenen und intersektionalen Begriff von Feminismus. Er richtet sich gegen die Enthierarchisierung, Liberalisierung und Entnormierung von Geschlechterverhältnissen. Spezifisch für ,Gender'-Kritiker_innen ist der Fokus auf die mit dem Begriff Gender verbundene (und von ihnen abgelehnte) Anerkennung einer Vielfalt geschlechtlicher und sexueller Identitäten. Insofern sind sie eigenständig zu betrachten. Die Ursachen der vermeintlichen Probleme sehen sie in Emanzipation, Frauenbewegungen und Feminismen. Insofern sind sie auch als antifeministisch zu bezeichnen.

Daran anschließende Diffamierungen wie ,Gender-Wahn` oder ,Gender-Gaga ‘ sind in den vergangenen Jahren weit ins konservative und liberale Spektrum eingesickert, wie auch die eingangs erwähnten Schlagzeilen nahelegen. Sie weisen ein hohes Mobilisierungs- und Radikalisierungspotenzial auf (Aigner 2013; Lang/Peters 2018; Vorländer/Schäller/Herold 2016). So machte sich die CDU-Vorsitzende Annegret Kramp-Karrenbauer in einer Büttenrede beim Karneval 2019 über die Einrichtung von All-gender-welcome-Toiletten lustig (u.a. rbb24 2019). Der Aufruf „Schluss mit dem Gender-Unfug“, den der Verein Deutsche Sprache gegen einen genderbewussten Sprachgebrauch im März 2019 lancierte, wurde auch von Journalist_innen, Schriftsteller_innen, Publizist_innen und Autor_innen unterschrieben, die sich selbst nicht als konservativ bezeichnen würden (Verein Deutsche Sprache 2019). Lang (2015) spricht von einer Brücken- oder Scharnierfunktion, Kováts und Põim (2015) von Gender als „symbolic glue“, der Bündnisse der genannten Gruppierungen bis in konservative, traditionalistische und sogar liberale Kreise ermöglicht. Mit dem Erstarken einer sogenannten Neuen Rechten nimmt die Kritik an Gender-Politiken und -Diskursen auch europaweit zu (vgl. Kováts/Põim 2015; Kuhar/Paternotte 2017). Den Anschein wissenschaftlicher Fundierung erhalten diese

überwiegend von Kritiker_innen benutzt, um den Begriff Gender in die Nähe von Ideologie und totalitärem Denken zu rücken. Für kritisch-wissenschaftliche Bezugnahmen, so Scheele (ebd.), sei der Begriff, ,verloren“.

4 In Kombination mit dem Begriff Kritik setzte ich Gender in einfache Anführungszeichen, um die Zurückweisung dessen kenntlich zu machen, was eben diese Kritiker_innen mit dem Begriff verbinden und was nichts mit einem kritischen Diskurs über unterschiedliche Konzepte des Verhältnisses von Natur und Kultur gemein hat. 
Positionen auch durch wissenschaftliche ,Gender'-Kritiker_innen unter anderem aus der Evolutionsbiologie, die die vermeintliche Unveränderbarkeit der Geschlechterdifferenz behaupten (vgl. Näser-Lather in diesem Band).

Für die Funktion solcher Diskurse als Scharnier oder symbolischer Kitt sind politische Leitmedien ${ }^{5}$ zentral (Klaus 2008; Lenz 2011). In der Literatur werden einige Medienbeiträge aus dem Jahr 2006 als stilbildend oder prägend für ,gender'kritische Diskurse genannt (Frey 2014; Hark/Villa 2015; Lang 2015): Volker Zastrow, Politik-Chef der Frankfurter Allgemeinen Sonntagszeitung, polemisierte 2006 in drei Artikeln der FAZ gegen Gender Mainstreaming und Gender Studies (FAZ 24.05.2006, 19.06.2006, 07.09.2006) ${ }^{6}$ und fasste dies in einem Buch zusammen (Zastrow/Feuchtenberger 2006). Das zog in der extrem rechten Zeitung Junge Freiheit (07.07.2006) einen inhaltlich ähnlich ausgerichteten Artikel nach sich. Im Nachrichtenmagazin Der Spiegel (01/2007) folgte ein Artikel, in dem René Pfister Gender Mainstreaming als „Umerziehungsmaßnahme“ bezeichnete. Die genannten Autoren benutzten den Begriff ,Genderismus' zwar nicht selbst; jedoch wurde in der Rezeption beispielsweise durch die antifeministische Webseite Wikimannia eine polemische Abwertung feministischer Positionen mit diesem Label versehen (Frey 2014: 29, http://de.wikimannia.org/genderismus).

Dass es nicht in erster Linie ,gesellschaftliche Verlierer_innen' sind, bei denen ein vermeintlicher ,Anti-Genderismus', also die Kritik dessen, was als ,Genderismus' bezeichnet wird, Anklang findet, legen Untersuchungen von Vernetzungsstrukturen und politischen Positionierungen der Akteur_innen nahe (u.a. Billmann 2015; Kemper 2014a, b). Befunde zum sozioökonomischen Profil der Sympathisant_innen von AfD und Pegida weisen auf einen sehr hohen Männer- (ca. 80\%) sowie überdurchschnittlichen Akademiker_innen-Anteil hin; mit der AfD sympathisieren viele Einkommensstarke, mit Pegida viele Selbstständige (Berbuir/Lewandowsky/Siri 2015: 168; Daphi et al. 2015; Vorländer/Schäller/Herold 2016). Wimbauer/Mokatef/Teschlade (2015) interpretieren den Diskurs über ,Genderismus' daher als Abwehr gegen multiple gesellschaftliche Verunsicherungen. Sie führen in

5 Der Begriff Leitmedium ist in der Medienwissenschaft umstritten. Einerseits scheint es einen impliziten Konsens über politische Leitmedien zu geben (Blum 2011: 7). Kriterien seien die von Leser_innen zugeschriebene Glaubwürdigkeit, Kompetenz und publizistisch-journalistische Relevanz, eine explizierte normative redaktionelle Grundhaltung und die Fähigkeit, gesellschaftliche Diskurse zu beeinflussen (Jarren/Vogel 2011: 23f). Andererseits repräsentieren diese Kriterien den hegemonialen Anspruch einer einzigen (androzentrischen) politischen Öffentlichkeit (Beck 2016: 81-83). Aus forschungspragmatischen Gründen arbeite ich dennoch mit diesem Begriff.

6 Die genauen Titel der zitierten Medienbeiträge sind am Ende des Literaturverzeichnisses unter „Primärquellen: Text-Sample“ aufgelistet. 
erster Linie die von der rot-grünen Bundesregierung (1998-2005) begonnenen arbeitsmarkt- und familienpolitischen Reformen an. Darüber hinaus ist zu vermuten, dass auch gleichstellungs- und geschlechterpolitische Reformen (v.a. Gender Mainstreaming, eingetragene Partnerschaften, Sukzessiv-Adoption für eingetragene Paare, dritte Kategorie im Personenstandsrecht, vielfaltsorientierte Reformen von Bildungsplänen) zur Verunsicherung androzentrischer Normativitätsvorstellungen beitrugen.

Diese Befunde wie auch eine Medienanalyse von Aigner (2013) implizieren, dass öffentliche Diskurse über ,Genderismus' vor allem von konservativen Teilen der oberen Mittelschicht getragen werden. Um der Bedeutung von politischen Medien als Diskursarenen Rechnung zu tragen, wurden in dem Projekt ,Genderismus ' in der medialen Debatte. Themenkonjunkturen 2006 bis $2016^{7}$ exemplarisch Medien mit einer Leser_innenschaft untersucht, deren Profil auf eine Offenheit für ,Genderismus'-Diskurse schließen lässt. Dem wurden Medien gegenübergestellt, bei denen eine größere Liberalität vermutet wurde. Das Projekt ging der Frage nach, ob es Thematisierungskonjunkturen entsprechender Mediendiskurse im Zeitverlauf gibt und worauf diese gegebenenfalls zurückzuführen sind. Daneben wurden Deutungen von Gleichstellungspolitiken, Gender Mainstreaming und Geschlechterforschung in den Medientexten sowie in Kommentaren von Leser_innen untersucht. Der Untersuchung lag die These zugrunde, dass sich antifeministische Diskurse in Reaktion auf solche staatlichen Reformen und Liberalisierungsmaßnahmen, politischen Forderungen und gesellschaftspolitischen Ereignisse verdichten, die androzentrische Normativitätsvorstellungen in Frage stellen und damit Verunsicherung auslösen. Ein Augenmerk lag auch auf Anschlüssen an die Fallstudien des REVERSE-Projekts. Das Projekt, Genderismus ' in der medialen Debatte ist damit eine erste Langzeituntersuchung der Bedeutung politischer (Leit-)Medien für ,gender'-kritische gesellschaftliche Diskurse.

Untersucht wurden Diskursbeiträge in den beiden konservativen Tageszeitungen Frankfurter Allgemeine Zeitung (FAZ) und Die Welt. Zwei liberale Medien, die Tageszeitung Süddeutsche Zeitung (SZ) und der Nachrichtendienst Spiegel online (SPON) als das intermediale Leitmedium ${ }^{8}$ schlechthin (Netzwerk Recherche 2006) dienten dem Vergleich. Die Beschränkung auf nur vier Medien ermöglichte es, den langen Zeitraum von elf Jahren abzudecken, jedoch mit zwei Einschränkungen. Zum einen bringt es die Eingrenzung auf sogenannte Print-Leitmedien (Jarren/

7 Das von mir bearbeitete Projekt, Genderismus ' in der medialen Debatte wurde vom Hessischen Ministerium für Wissenschaft und Kunst gefördert und hatte eine Laufzeit vom 01.08.2017 bis 31.01.2019.

8 Der Begriff intermediales Leitmedium weist darauf hin, dass sich andere Nachrichtenmedien in hohem Maße an der Themensetzung von Spiegel online orientieren. 
Vogel 2011: 23f) mit sich, dass die Befunde nicht ohne Weiteres verallgemeinert werden können, sondern in anderen Medien weiter überprüft werden müssen. Zum anderen besteht die Möglichkeit, dass wegen der Beschränkung des Samplings auf den Begriff Gender thematisch passende Diskursbeiträge nicht berücksichtigt wurden, in denen dieser Begriff nicht genannt wurde.

Das Sample bestand aus knapp 1.000 Artikeln (FAZ: 355, Welt: 273, SZ: 172, SPON: 144). Es wurde anhand von häufig genannten Gender-Komposita gezogen. ${ }^{9}$ In vier Stufen wurden die Texte einer quantitativen Inhaltsanalyse, einer strukturierenden qualitativen Inhaltsanalyse, einer Frame-Analyse ausgewählter Artikel und einer qualitativen Inhaltsanalyse von darauf bezogenen Kommentaren von Leser_innen unterzogen. Die Gliederung des vorliegenden Beitrags folgt diesem Aufbau. Dabei möchte ich die These plausibilisieren, dass politische Medien überwiegend unabhängig von externen Ereignissen Diskursbeiträge veröffentlichen und damit als eigenständige Akteur_innen in diesem gesellschaftlichen Diskurs auftreten. Zugleich wird die widersprüchliche Bandbreite politischer Positionierungen zwischen den Medien, aber auch innerhalb der untersuchten Titel diskutiert, von der bereits erwähnten Scharnierfunktion der, Gender'-Kritik über Diskurse von vermutlich begrenzter gesellschaftlicher Reichweite bis hin zu Texten, die eine weitgehende Akzeptanz gesellschaftlicher Vielfalt erkennen lassen.

\section{Im FokUs der Medien: Gender Mainstreaming UND GESCHLECHTERFORSCHUNG}

Der Begriff Gender wird in den Medien häufig genannt. Der Suchbegriff Gender ergab im Untersuchungszeitraum von 2006 bis 2016 Tausende Treffer, von denen die meisten jedoch nichts mit der, Genderismus'-Debatte zu tun hatten. Deswegen wurde die Artikel-Suche auf Komposita mit dem Begriff Gender eingeschränkt. ${ }^{10}$

9 Wortstämme $\left(^{*}\right)$ und unterschiedliche Schreibweisen folgender Gender-Komposita: Gender-Agenda, Gender-Aktivis*, Gender-Beweg*, Gender-Debatte, Gender-Diskurs, Gender-Forsch*, Gender-Fragen, Gender-Gaga, Gendergerecht*, Gender-Ideologie, Genderisierung, Genderismus, Gender Mainstreaming, Gendern bzw. gegendert, Gender-Politik, Gender-Revolution, Gender-Sprache, Gender Studies, Gender-Thema, Gender-Theor*, Gender Trouble. Die Suche wurde mit Komposita begonnen, die in der Literatur vorgefunden wurden. Diese wurden sukzessiv induktiv erweitert.

10 Ich danke Katharina Aleksin, Christina Alicia Bernhard, Melanie Kuhnert und Armin Sauermann, die als studentische Hilfskräfte an der Erstellung des Text-Korpus mitwirkten. 
Die quantitative Inhaltsanalyse ergab, dass die am häufigsten genannten Begriffe im Sample Gender Mainstreaming (191 Texte) und Gender Studies (190) sind. Bei Gender Mainstreaming gibt es ein starkes Gefälle von der FAZ (91) über die Welt (72), SPON (20) zur SZ (8). Bei Gender Studies sind die Unterschiede erheblich geringer (FAZ 58, Welt 54, SPON und SZ je 39). Auf den Plätzen drei und vier folgen die Begriffe Gender-Theor* (139 insgesamt, FAZ 72, Welt 32, SPON 17, SZ 18) und Gender-Forsch* (122 insgesamt, FAZ 51, Welt 28, SPON 24, SZ 19). Neben dem Konzept des Gender Mainstreaming wurden in den untersuchten Medien also am häufigsten Gender-Komposita genannt, die auf Diskurse über die wissenschaftliche Auseinandersetzung mit Gender schließen lassen. ${ }^{11}$

Zwischen dem Konzept Gender Mainstreaming sowie den wissenschaftsbezogenen Komposita an der Spitze und weiteren Begriffen klafft eine Lücke. Der Begriff Gender-Fragen an nächster Stelle wurde 82-mal genannt, Gendern/gegendert und Gender-Debatte je 54-mal. Der mit 35 Nennungen häufigste polemisierende und diffamierende Begriff ist Gender-Ideologie. Auch hier gibt es einen großen Unterschied zwischen den konservativen Blättern FAZ (13) und Welt (14) sowie den liberalen Titeln SPON (6) und SZ (2). Weitere stark abwertende, polemische oder ideologisch aufgeladene Begriffe wie Gender-Agenda, Gender-Aktivis*, Genderisierung, Gender-Gaga, Genderismus, Gender-Revolution, Gender-Verschwörung und Gender-Wahn wurden vergleichsweise wenig genannt (FAZ: 17, SPON: 17, Welt: 4, SZ: 4).

Auch wenn die in der Literatur hervorgehobenen Medienbeiträge, die sich abwertend oder polemisch zu Gender Mainstreaming oder Geschlechterforschung äuBerten (FAZ 19.06.2006, Junge Freiheit 07.07.2006, Der Spiegel 01/2007) als stiloder diskursprägend qualifiziert werden, haben sie in den untersuchten Medien auf der Ebene der dort verwendeten Begriffe keine größere Resonanz hervorgerufen. Außerdem wurden Gender-Thematiken bereits erheblich früher medial verhandelt, weit überwiegend in der FAZ. Anlässe waren vor allem die UN-Bevölkerungskonferenz 1994 in Kairo und die UN-Frauenkonferenz 1995 in Peking (vgl. Kuhar/ Paternotte 2017; Näser-Lather 2019: 111). Daneben war der erste rot-grüne Koalitionsvertrag 1998, in dem Gender Mainstreaming als Instrument festgeschrieben wurde, ein solcher Anlass.

Die Häufigkeit der Nennung von Gender-Komposita nimmt im Zeitverlauf erheblich zu. Allerdings weichen einzelne Medien und einzelne Begriffe teils stark davon ab. Der Trend ergibt sich vielmehr in der Summe. Von tatsächlichen Verläufen kann nur bei den Begriffen Gender Mainstreaming, Gender Studies, GenderTheor*, Gender-Forsch*, Gender-Fragen und Gender-Debatte gesprochen werden.

11 Für den Begriff Gender-Theor* gilt dies eingeschränkt. Auch in anderen Kontexten wird von Gender-Theorie gesprochen, wie die Einleitung zum vorliegenden Beitrag zeigt. 
Die Summe der auf Wissenschaft bezogenen Begriffe lässt darauf schließen, dass neben dem Instrument Gender Mainstreaming insbesondere die Geschlechterforschung im Fokus der Medien stand.

\section{Die Medien als eigenständige Akteure IN ,GENDERISMUS'-DEBATTEN}

Thematisierungen, in die Gender-Komposita eingebettet waren, wurden in einer qualitativ-strukturierenden Inhaltsanalyse (Mayring 2010; Meyen et al. 2011) untersucht. Als Hauptkategorien dienten die Codes Anlass, Thema und Erwähnung (für Texte ohne thematischen Bezug). Anlass und Thema wurden induktiv immer weiter ausdifferenziert.

In den untersuchten Medien wurde der Begriff Gender häufig ohne thematische Einbindung genannt. So wurden beispielsweise Expertinnen in unterschiedlichen Kontexten als Gender-Forscherin vorgestellt oder der Kampf gegen das Gender Mainstreaming als Programmatik der AfD aufgezählt, ohne dass darauf thematisch im Artikel weiter eingegangen wurde. Diese Texte wurden gestrichen.

Abbildung 1: Häufigkeit der Thematisierung gender-bezogener Fragen in den untersuchten Medien.

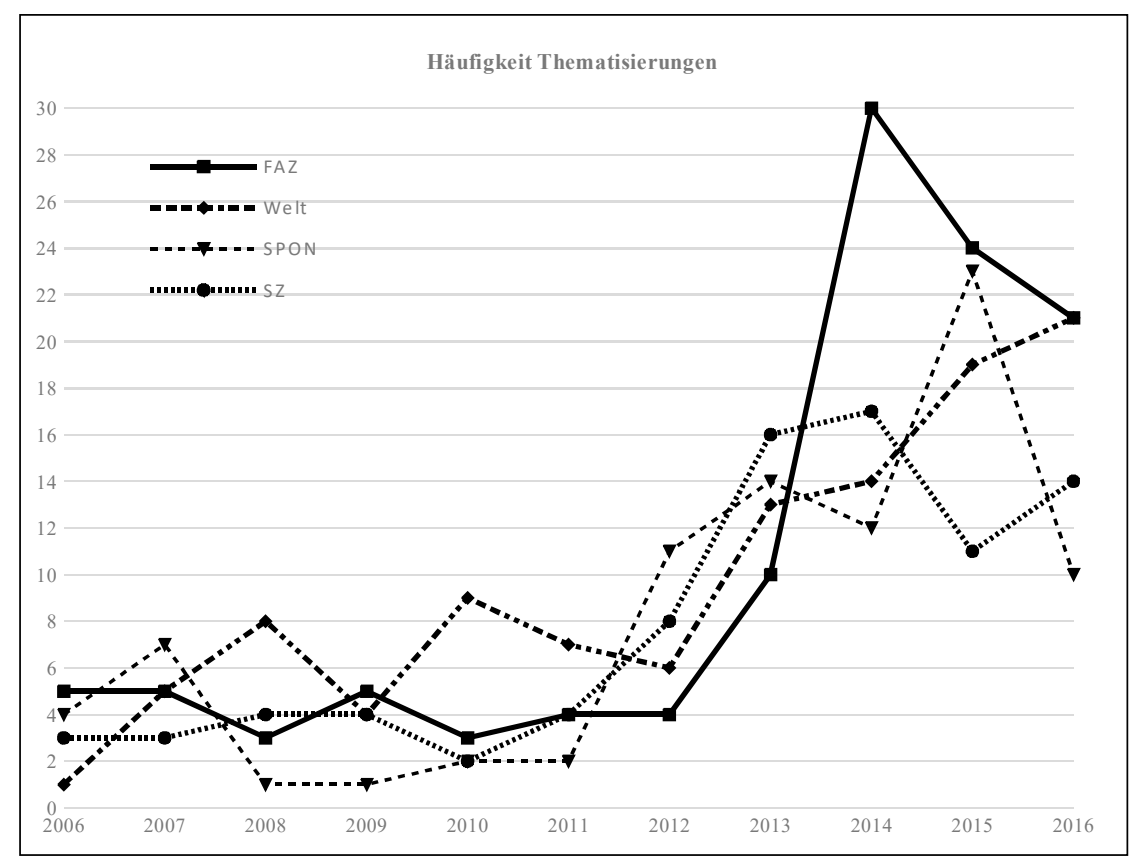

Quelle: Eigene Berechnung. 
Der Verlauf der Summe aller Thematisierungen im gesamten Zeitraum rechtfertigt die Bezeichnung Konjunktur. Allerdings haben die bereits genannten diskursprägenden Beiträge 2006/2007 kaum eine gehäufte thematische Bezugnahme auf Gender nach sich gezogen. Die von Frey (2014), Hark und Villa (2015), sowie Lang (2015) konstatierte Wirkung bezieht sich vor allem auf die argumentative Ebene. Für die quantitativen Befunde des exemplarischen ,Genderismus'-Projekts gilt diese Aussage hingegen nicht.

Von gehäuften Thematisierungen mit Gender-Bezug lässt sich erst ab 2011 bis 2013 sprechen. Dieser starke Anstieg zeugt von einer Intensivierung der medialen Debatte. Allerdings weichen einzelne Medien und Begriffe von dieser Gesamtkurve ab, die gleichsam in einem medienübergreifenden Diskurs entsteht (siehe Schaubild). Dennoch gibt es so gut wie keine gemeinsamen anlassbezogenen Thematisierungen. Die Medien berichten nicht in erster Linie aus Anlass bedeutender externer Ereignisse, sondern setzen eigene Themen und lassen dazu Redaktionsmitglieder wie auch (namhafte) Gastautor_innen zu Wort kommen. Sie treten also als eigenständige Akteure in diesem Diskurs auf.

Zwar nimmt die Zahl der Thematisierungen mit Gender-Bezug parallel zum Aufstieg der AfD, die 2013 gegründet wurde, und von Pegida ab 2014 zu. Der Anstieg beginnt jedoch schon früher, nämlich ab 2011. Und er hat nichts mit der Berichterstattung über die $\mathrm{AfD}$ zu tun, auch wenn in so manchem Artikel über diese Partei eine Erwähnung des Begriffs Gender-Wahn nicht fehlen darf (u.a. Welt 27.01.2016). Dass Gender-Komposita im Kontext der AfD zwar 36-mal erwähnt werden, hauptsächlich in der FAZ und der Welt, jedoch nur fünfmal thematisch eingebettet werden, plausibilisiert diesen Befund. Eine Erklärung könnte sein, dass die FAZ und die Welt der AfD zwar genderpolitisch nahestehen, in der Auseinandersetzung mit der AfD Geschlechterverhältnisse jedoch nicht unbedingt als relevant angesehen werden.

Während medienübergreifende anlassbezogene Thematisierungen fehlen, sind thematische Gemeinsamkeiten ohne Bezug zu einem externen Ereignis und ohne zeitlichen Zusammenhang erkennbar, auch wenn nicht jedes Medium jedes Thema in gleichem Umfang verhandelte. Herausgehobene Bedeutung haben Problematisierungen von Gender Studies, genderbewusster Sprache, von Männerrechten und Männlichkeit(en), von queeren Rechten, Gender und Islam sowie von Feminismus und vermeintlichen Auswüchsen des Gender Mainstreaming.

Daneben hatte jedes Medium je eigene thematische Schwerpunkte. Die FAZ verhandelte ausführlich die vielfaltsorientierte Reform der Bildungspläne in BadenWürttemberg und Hessen sowie die teils gewalttätigen Proteste gegen geschlechtliche Vielfalt in Frankreich. Die Welt thematisierte Gender in Bezug auf den Islam, und zwar bereits vor den Übergriffen in der Kölner Silvesternacht 2015. In der SZ wurden im Streiflicht, der Kolumne auf Seite 1, immer wieder Geschlechter-Kli- 
schees und Stereotype glossiert und teils aktualisiert. Auf SPON gab es hingegen keine erkennbaren thematischen Schwerpunkte, wohl aber veröffentlichte Jan Fleischhauer als konservatives Aushängeschild des Online-Dienstes immer wieder ,gender'-kritische Kolumnen.

Artikel, die sich auf die genannten zeitlich voneinander unabhängigen medienübergreifenden Themen bezogen, sowie Artikel, die zu den medienspezifischen Schwerpunkten gehörten, wurden einer Frame-Analyse unterzogen, um typische Argumentationsmuster, rhetorische Figuren und Stile sowie deren Rezeption durch die Leser_innen herauszuarbeiten.

\section{Medien-Frames zu Gender: ZWISCHEN ANGRIFF UND AKZEPTANZ}

Unter Framing wird das aktive und selektive Hervorheben von Informationen und Positionen durch politische Akteur_innen, durch die Autor_innen von Medienbeiträgen sowie durch die Rezipierenden verstanden (Matthes 2014: 10f). Frames sind das Ergebnis dieses Prozesses und werden im Verlauf eines Diskurses durchgesetzt oder neu justiert, um öffentliche Aufmerksamkeit zu erlangen (Matthes 2014: 15). Matthes (2014: 10) begreift Frames als „,Sinnhorizonte“ von Akteuren [...], die gewisse Informationen und Positionen hervorheben und andere ausblenden." Anhand von Frames können Deutungen und Sinngebungen in den Medientexten beschrieben werden. Diese Methode eignet sich daher besonders dafür, Argumentationsund Deutungsmuster in ,Genderismus'-Diskursen jenseits der manifesten Ebene herauszuarbeiten.

Für die Frame-Analyse wurde das Sample aus der Untersuchung der Thematisierungsverläufe thematisch weiter konzentriert. Einbezogen wurden die Schwerpunkte vielfaltsorientierte Pädagogik (FAZ) sowie Gender und Islam (Welt), ergänzt um thematisch passende Artikel der anderen Medien. Daneben wurden die medienübergreifenden Thematisierungen ohne zeitlichen Zusammenhang (Gender Studies, genderbewusste Sprache, Männerrechte/Männlichkeit(en), queere Rechte und Feminismus/vermeintliche Auswüchse des Gender Mainstreaming) herangezogen. Berücksichtigt wurden nur Beiträge, deren Länge eine Frame-Analyse überhaupt zuließ. Diese Reduktionsstrategie ergab ein Sample von 59 Artikeln (FAZ 15, SPON 14, SZ 14 und Welt 16). Untersucht werden Frames in einer qualitativen Inhaltsanalyse anhand der Hauptkategorien Problemdefinition, Ursachenzuschreibung, Bewertung und Lösungsvorschläge, die sich als effizient für die Frame-Analyse durchgesetzt haben (Entman 1993).

Insgesamt wurden neun Frames identifiziert und größtenteils in Frame-Gruppen eingeteilt: 
- Die Frames Bedrohungs-Szenarien, Dominanz einer Minderheit über die Mehrheit und Unterwerfungs-Szenarien wurden zur Frame-Gruppe Angriffe auf Heteronormativität zusammengefasst.

- Der Frame Regeln einer demokratischen Öffentlichkeit steht thematisch mit der genannten Frame-Gruppe in Verbindung.

- Die Frames Gender Studies als Pseudowissenschaft und politische Beeinflussung durch Sprache bilden die Frame-Gruppe Vorwurf der politischen Instrumentalisierung von Wissenschaft.

- Der Frame Hinterfragung von Männlichkeiten umfasst unterschiedliche Diskurse in mehreren zeitlichen Phasen.

- Der Frame-Gruppe Wertschätzende Diskurse über geschlechtliche Vielfalt umfasst die zwei Frames Spiel mit Geschlecht im Feuilleton, in dem Kunst- oder Kulturthemen in Gender-Perspektive verhandelt wurden, sowie Verständnis für Einzelschicksale mit Features über homosexuelle und trans-idente Menschen.

Sieben Texte konnten keinem übergreifenden Frame zugeordnet werden. Mit 15 Texten ist die Frame-Gruppe Angriffe auf Heteronormativität quantitativ am bedeutendsten. Dies tritt umso deutlicher zu Tage, wenn man die drei sich inhaltlich hier einfügenden Texte des Frames Regeln einer demokratischen Öffentlichkeit hinzuzählt. Die anderen Frame-Gruppen und der Frame Hinterfragung von Männlichkeiten sind mit zwölf beziehungsweise zehn Texten annähernd gleichgewichtig.

\subsection{Frame,Angriffe auf Heteronormativität‘}

Die Texte der Frame-Gruppe Angriffe auf Heteronormativität stellen die zweigeschlechtliche Ordnung in unterschiedlichen Aspekten in Frage, teils kombiniert mit Problematisierungen der Konsequenzen der Zuwanderung aus anderen Kulturkreisen.

Der Bedrohungs-Frame umfasst sieben Artikel. Fünf verhandeln das Thema geschlechtliche und sexuelle Vielfalt in pädagogischen Kontexten und bilden damit den Kern des Frames. Dieses Thema kam nicht erst mit den Protesten gegen den neuen Bildungsplan in Baden-Württemberg 2014 auf, auch wenn dieser Konflikt am meisten Gewicht hat. Bereits 2007 kritisierte der Soziologe Gerhard Amendt einen Elternratgeber zur Sexualaufklärung aus der Zeit der rot-grünen Bundesregierung, den Familienministerin Ursula von der Leyen (CDU) zurückgezogen hatte (Welt 26.10.2007, vgl. Abschnitt 5.2). In der FAZ wurden 2011 Unterrichtsmaterialien zum Thema geschlechtliche Vielfalt und Homosexualität in Berlin kritisiert (FAZ 20.07.2011). Zwei weitere Artikel verhandelten eine Demonstration gegen die Ehe für alle in Frankreich (Welt 03.06.2013) und Shitstorms von Männern gegen erfolgreiche Frauen (SZ 27.08.2016). 
Der vielfaltsorientierte Bildungsplan in Baden-Württemberg wurde als Bedrohung für Kinder, Jugendliche und Familien problematisiert. Er fördere Kindesmissbrauch, verleite Kinder und Jugendliche zum Sex, entnaturalisiere oder entnormalisiere die traditionelle Familie aus Vater, Mutter und Kind(ern) (FAZ 23.10.2014). Als Verursacher_innen der Bedrohung machte der Soziologe Gerhard Amendt, diesmal in der FAZ (31.12.2014), homosexuelle oder vermeintlich psychisch gestörte Menschen aus, die , in entscheidenden Phasen ihrer psychosexuellen Erfahrungen während der Kindheit" an einer problematischen oder fehlenden Beziehung zu den Eltern gelitten hätten. In einem weiteren Artikel (FAZ 23.10.2014) klingen verschwörungstheoretische Muster an: Im Konflikt um den Bildungsplan in BadenWürttemberg hätten Eltern erst durch eine Online-Petition von der mit dem neuen Plan drohenden Frühsexualisierung und Umerziehung erfahren. Nur in der SZ wurde der Bedrohungs-Frame distanzierend zugespitzt. Kinder sollten lernen, dass neben Heterosexualität auch Homosexualität und anderes okay sei. „192.000 Menschen [...] haben eine Online-Petition dagegen unterschrieben. Ihre [...] Exegese des Papiers legt offenbar nahe, dass Kindern künftig gleichgeschlechtliche Liebe mit Pornos schmackhaft gemacht werden soll." (SZ 04.02.2014)

Im Frame Dominanz einer Minderheit über die Mehrheit (vier Texte) wurde das Verhältnis von Mehrheit und Minderheit problematisiert. In einem FAZ-Text (FAZ 27.06.2015) ging es um eine ,schweigende Mehrheit“ gegen gleichgeschlechtliche Partnerschaften in Italien. In zwei Welt-Artikeln (Welt 14.06.2013, 26.06.2015) wurde eine homosexuelle Minderheit imaginiert, die die Macht über die heterosexuelle Mehrheit übernehmen wolle. In einem weiteren Text der gleichen Zeitung (Welt 23.06.2015) hieß es, die grün-rote Regierung in Baden-Württemberg wolle einen Aktionsplan zum Schutz sexueller Minderheiten undemokratisch am Parlament vorbei verabschieden und befasse sich mit dem Sex von Minderheiten statt mit wirklich wichtigen Problemen. Kern des Frames ist also eine Minderheit, die der Mehrheit ihren Willen aufzwingen wolle.

Als Ursache beziehungsweise Verursacher_innen wurden mal eine ,ideologische Avantgarde“ im Milieu der emanzipierten Minderheiten (Welt 14.06.2013), mal die „Gender-Diktatur“ oder „Genderpflicht“ (Welt 26.06.2015) genannt. Beides suggeriert die Missachtung demokratischer Freiheiten. Schüler_innen in BadenWürttemberg würden im Stil der DDR umerzogen. Dies klingt in Bewertungen wie „Korrektheitskämpfer“, „Ideologie der Minderheitenmacht“, „oktroyierte Sexualvielfalt", „Sexualpioniere“, ,gedankenpolizeilicher Schub“ oder „Emanzipationskommissarinnen und -kommissare“ (Welt 26.06.2015) an.

Der Frame Unterwerfungs-Szenarien war in vier Artikeln zu identifizieren. In drei Artikeln ging es um die Unterwerfung unter den Feminismus. In einem Artikel über eine neue Männerrechtsbewegung (FAZ 16.10.2015) wurde argumentiert, Gleichstellungspolitik und Gender Mainstreaming benachteiligten Männer und Jungen systematisch. Als Beweis dienten erhöhter Drogenkonsum, Suizidalität, Ar- 
beitslosigkeit und Obdachlosigkeit von Männern, aber auch der Verlust des Sorgerechts für die Kinder nach einer Scheidung. Dies impliziert die Bewertung, diese Probleme würden gesellschaftlich weder erkannt noch bekämpft, während für Frauen alles getan werde. Auf SPON (04.07.2013) wurde die Entscheidung der Universität Potsdam für das generische Femininum in ihrer Grundordnung mit angeblich drohendem Ärger durch Feministinnen begründet. Und in einer Sammelrezension dreier Feminismus-Bücher in der Welt (28.06.2014) wurden Regeln, Verbote sowie ,richtige“ Sprache und Theorie im Feminismus als Problem benannt. In einem Artikel in der Welt (12.04.2016) über eine Gesetzesinitiative zum Verbot von sexistischer Werbung fiel der Begriff „Unterwerfung“ explizit. Sexistische Werbung wurde nicht als Sexismus, sondern als „Erotik in der Öffentlichkeit“ verhandelt, die eliminiert werden solle. Wie im Frame Dominanz einer Minderheit über eine Mehrheit hat das Narrativ etwas mit undemokratischen Praktiken zu tun. Allerdings wird hier angeprangert, ein als Ideologie diffamierter Feminismus habe die Änderung oktroyiert.

Die Ursache der Probleme wurde in allen Artikeln dem Druck oder der Macht von Feministinnen oder von Gleichstellungs- beziehungsweise Gender-Politiken zugeschrieben. Diese Argumentationsfiguren finden sich bereits bei Zastrow (Zastrow/Feuchtenberger 2006). So wurde bemängelt, die Kommission zum Zweiten Gleichstellungsbericht der Bundesregierung sei größtenteils mit Feministinnen und Vertreterinnen der Gender Studies besetzt (FAZ 16.10.2015). In der Sammelrezension fiel der Begriff „Geschlechtsbesessenheit“ (Welt 28.06.2014). Der Entwurf zum Verbot sexistischer Werbung wurde als „Zensurgesetz“ verurteilt. Dabei wurde ein Bezug zur Kölner Silvesternacht 2015 hergestellt, indem sexistische Werbung ironisch als Ursache für die Übergriffe bezeichnet wurde (Welt 12.04.2016).

Alle drei Frames der Gruppe Angriffe auf Heteronormativität haben als Fluchtpunkt eine zweigeschlechtliche und geschlechtshierarchische Normalität. Allerdings unterscheiden sich die Perspektiven. Im Zusammenhang mit Kindern und Jugendlichen wird geschlechtliche Vielfalt als Bedrohung inszeniert. Dabei dient die Chiffre des unschuldigen, asexuellen Kindes als vermeintlicher moralischer Kompass (Schmincke 2015). Als Bedrohungskulissen werden Homosexualität, psychische Störungen und vermeintliche Verschwörungen aufgebaut. Der Frame Dominanz einer Minderheit über die Mehrheit wird herangezogen, um die rechtliche und gesellschaftliche Anerkennung geschlechtlicher Vielfalt insgesamt zurückzuweisen. Queere Rechte werden als Beschneidung von Freiheitsrechten einer zweigeschlechtlichen Mehrheit imaginiert. Im Frame Unterwerfungs-Szenarien dominiert der Ideologie-Vorwurf. Feminismus als Ideologie wolle mit Ge- und Verboten Menschen gängeln, ihre Individualität und ihre Freiheiten beschneiden. Diese Frames entstammen ursprünglich christlich-fundamentalistischen, neu-rechten und maskulistischen Argumentationsmustern (u.a. GEW 2016). Medien machen sich diese Narrative teilweise zu eigen, besonders dann, wenn sie Gastautor_innen, die 
diesen Kreisen zuzurechnen sind, mit moralisch aufgeladenen Essays zu Wort kommen lassen.

Die Feststellung, Medien setzten ihre Themen im ,Genderismus'-Diskurs eigenständig und richteten sich nicht nach externen Anlässen, gilt für die Frame-Gruppe Angriffe auf Heteronormativität tendenziell weniger. Ereignisse und Prozesse, auf die sich diese Medientexte beziehen, waren vor allem die Proteste gegen den vielfaltsorientierten Bildungsplan in Baden-Württemberg (2013) und Auseinandersetzungen um queere Rechte.

\subsection{Frame, Regeln einer demokratischen Öffentlichkeit‘}

Drei Texte über die Debatte zur Integration von Zugewanderten und über queere Rechte, die eine inhaltliche Nähe zur oben beschriebenen Frame-Gruppe aufweisen, wurden als Regeln einer demokratischen Öffentlichkeit gerahmt, zwei in der Welt (28.12.2015, 23.05.2016) und einer in der FAZ (18.08.2016). Auch hier wurde das Verhältnis einer gesellschaftlichen Mehrheit oder Normalität zu etwas Anderem thematisiert. Allerdings war der Tenor ein komplett anderer, weil anhand der Konstruktion des Anderen die Regeln der Mehrheitsgesellschaft diskutiert wurden. Thema waren also nicht die (,verwerflichen') Intentionen der Anderen, sondern die Regeln, an die diese sich zu halten hätten. Dies enthält den Aspekt des demokratischen Diskurses und ist nicht durchweg negativ konnotiert. Aus diesem Grund wurde der Frame nicht in die oben genannte Gruppe integriert.

Der CDU-Politiker Jens Spahn problematisierte in einem Kommentar zum Burka-Verbot (FAZ 18.08.2016) die Vollverschleierung als Isolation von einer demokratischen Gesellschaft. Das sei das Gegenteil von Offenheit und Öffentlichkeit, Deliberation und Teilhabe. Der Historiker Andreas Rödder begriff in einem Gastbeitrag in der Welt (28.12.2015) Zuwanderung als Frage an die gesellschaftliche „Hausordnung“. In einem Artikel über den ersten Queer-Kongress der Grünen (Welt 23.05.2016) wurden spezielle Diskurse und Besonderheiten der queeren Community als Hindernis für den Dialog mit der Mehrheitsgesellschaft begriffen.

Rödder zeichnete die Entwicklung der gesellschaftlichen „Hausordnung“ vom Gegensatz des „freien Westens“ zum „Zwangskollektivismus“ des Ostens über den „shareholder-value-Kapitalismus“ nach, der in der Finanzkrise 2008 einen Rückschlag erlitten habe (Welt 28.12.2015). Auf die Finanzkrise sei eine „Kultur der Inklusion“ gefolgt, ,die Emanzipation des Randständigen und der Nachteilsausgleich für vormals Benachteiligte - Frauen, Menschen mit Behinderungen, sexuell anders Orientierte“. Dies sei ,durch Gender-Mainstreaming und Antidiskriminierungsgesetz im Top-down-Verfahren implementiert“ worden und habe ,zugleich hart ideologische Züge“" gewonnen (ebd.). Damit nahm er Anleihen an den Ideologie-Vorwurf des Unterwerfungs-Frames. Man könnte also interpretieren, die Ideologie habe 
(wie schon im Kalten Krieg) die Oberhand gewonnen. Doch anders als Spahn, für den die Regeln der demokratischen Öffentlichkeit klar waren, betonte Rödder, gesellschaftliche Werte seien „stets Gegenstand der Aushandlung und von Machtkonflikten - und ständig in Bewegung“. Er empfahl eine Selbstverständigungsdebatte, in der auch „Überregulierungen der Gleichstellungspolitik“ hinterfragt werden könnten (ebd.).

\subsection{Frame-Gruppe ,Vorwurf der politischen Instrumentalisierung von Wissenschaft'}

In der Frame-Gruppe Vorwurf der politischen Instrumentalisierung von Wissenschaft sind die Frames Gender Studies als Pseudowissenschaft (sieben Texte) und politische Beeinflussung der Sprache (fünf Texte) versammelt. Dabei werden Geschlechterforscher_innen auch als Verursacher_innen einer sprachlichen Verunstaltung gekennzeichnet.

Der Frame Gender Studies als Pseudowissenschaft tritt in drei sich überlappenden Ausprägungen auf: Gender Studies als Ideologie, als quasi-religiöser Eifer und als Spinnerei/Blödsinn/Gender-Wahnsinn. Alle drei Ausprägungen beziehen sich auf Zweifel an der Wissenschaftlichkeit der Gender Studies und auf die dort weithin praktizierte genderbewusste Sprache. Der Tenor ist fast durchweg extrem abwertend bis polemisch. Eine ernsthafte Auseinandersetzung mit der Disziplin ist kaum erkennbar. Soweit überhaupt auf wissenschaftliche Diskurse Bezug genommen wird, dienen evolutionsbiologische Perspektiven auf Geschlecht als weitgehend biologisch determiniert als Referenz.

Paradigmatisch für den Ideologie-Vorwurf ist ein Gastbeitrag des Frankfurter Biologie-Professors Hans Peter Klein in der FAZ (21.05.2015). Klein konstruierte eine Dichotomie aus Biologie als Wissenschaft und Gender Studies als Ideologie. Vermeintliche „Einstellungen, Mythen, Erzählungen oder Überzeugungen“ hier, „belast- und überprüfbare Fakten“ einer „modernen experimentell-analytischen Wissenschaft" dort. Dem liegt die Fehlinterpretation von Grundbegriffen der Geschlechterforschung zugrunde: Doing Gender, also die Aktualisierung der zweigeschlechtlichen Ordnung in der Interaktion (v.a. Goffman 1994; West/Zimmerman 1987), wird als Hinterfragung des biologischen Geschlechts im „Gender Doing“ erklärt, das politische Instrument Gender Mainstreaming als „Gleichberechtigung oder die Akzeptanz sexueller Vielfalt“ (FAZ 21.05.2015). ${ }^{12}$ In einem weiteren Arti-

12 Es wäre zu fragen, warum ein Wissenschaftler sich nicht mit anerkannten Definitionen der fraglichen Begriffe befasst, was redlicher wissenschaftlicher Arbeit entsprechen würde. Stattdessen werden Begriffe in einer Art und Weise missverstanden, die in höchstem Maße angreifbar machen (Krüger-Kirn et al. 2019; Näser-Lather in diesem Band). 
kel (FAZ 12.04.2014) wird der Ideologie-Vorwurf mit der Nähe der Gender Studies zum Feminismus, zur Politik sowie mit ihrer Entstehung aus der Frauenforschung und mit der Dogmatik eines ,gegenderten' Sprachgebrauchs begründet, was auf den Wunsch nach einer Neuordnung der Gesellschaft schließen lasse. Der dritte Artikel, der mit dem Ideologie-Begriff hantierte, ist eine Replik auf ein Interview mit dem umstrittenen, gender'-kritischen Evolutionsbiologen Ulrich Kutschera (SPON 12. 09.2015). Dieser hatte Gender Studies als religiösen Eifer diffamiert (SPON 04.09. 2015). Die Gastautorin und Sozialwissenschaftlerin Lilian Peter drehte den Ideologie-Vorwurf um. Die Gleichsetzung von Gender Studies und Kreationismus und die Setzung, nur die Biologie könne etwas über Geschlecht sagen, seien ihrerseits Ideologie.

In dem Interview hatte Kutschera zuvor die Gender Studies mit dem Kreationismus gleichgesetzt und als „quasi-religiöse Strömung unter der Tarnkappe des Gender Mainstreaming“ diffamiert, also die Frame-Ausprägung quasi-religiöser Eifer bedient. Grundlage des Textes waren neben Kutscheras Polemiken auch ablehnende Reaktionen des AStA und des Präsidiums der Universität Kassel. Doch während SPON mit Kutschera persönlich gesprochen hatte, wurden Hochschulleitung und AStA nur in schriftlichen Stellungnahmen zitiert. So konnte Kutschera deren „hinterhältige Vorgehensweise“ kritisieren, während eine Gegenrede fehlte. Damit ist dieser Artikel nicht nur ein Beispiel für die Figur des religiösen Eifers, sondern auch für mangelnde journalistische Sorgfalt.

Stilmittel der dritten Ausprägung des Pseudowissenschafts-Frames als Spinnerei, Blödsinn oder Gender-Wahnsinn waren beißende Ironie und die Kritik an aus dem Zusammenhang gerissenen Zitaten. So stellte SZ-Redakteur Christian Weber den Artikel „Krampfzone“ (SZ 16.04.2016) unter eine ironische Vorrede: „Vielleicht liegt es daran, dass der Autor dieser Zeilen ein weißer, cis*-positionierter Heterosexist ist, jedenfalls beschäftigt ihn die strukturelle Gewalt durch binär zwangsgegenderte öffentliche Klos eher wenig.“ (SZ 16.04.2016)

Zwar konstatierte Weber geringe Unterschiede zwischen Männern und Frauen in Anatomie und Hirn, konterkarierte diese Erkenntnis aber mit vermeintlichen evolutionsbiologischen Differenzen, affirmierte die Bewertung der Gender Studies als Spinnerei und ließ zugleich die Motive pseudo-religiöser Eifer und Ideologie anklingen:

„Eher kann man mit Jesuiten über die Existenz Gottes diskutieren als mit Gendertheoretikern über biologisch bedingte Geschlechtsunterschiede. Wer sie in der Community behauptet, wird exkommuniziert wie früher die Abweichler beim Kommunistischen Bund Westdeutschland.“ (SZ 16.04.2016)

Seine Assoziation führte der Autor mit einem als „,,Begriffsdrachen“ aus dem Gendersprech" bezeichneten, ohne Kontext wiedergegebenen Zitat fort und schloss dar- 
aus, die Gender Studies wollten sich nach Art alter Politsekten durch eine hermetische Sprache vor Kritik schützen (vgl. Abschnitt 5.1).

In einem weiteren Artikel in der Welt (19.11.2014) wurde die Analyse eines Rap-Songs in einem Gender-Studies-Seminar an der Humboldt Universität Berlin ins Lächerliche gezogen. Zwar nannte der Autor auch Beispiele für seiner Ansicht nach sinnvolle Geschlechterforschung, etwa in der Medizin oder zur Unterdrückung der Frau im Islam. Dennoch wurde den Gender Studies unterstellt, sie hielten Zweigeschlechtlichkeit für eine von weißen heterosexuellen Mittelschichts-Männern ersonnene Ideologie. In dieser Fehlinterpretation erscheint die Erkenntnis der sozialen und kulturellen Konstruiertheit von Geschlecht als völlig absurd.

Im Frame Politische Beeinflussung der Sprache haben bis auf eine Ausnahme alle Artikel einen ablehnenden Tenor. Die Kritik bezieht sich auf verschiedene Varianten einer genderbewussten oder genderneutralen Schreibweise, wie das BinnenI (SprecherInnen), der Asterisk (Sprecher*innen), der Gender_Gap (Sprecher_innen) oder die geschlechtsneutrale $\mathrm{x}$-Endung (Sprechx). Paradigmatisch für diese Problematisierungen ist ein Zitat des Sprachwissenschaftlers Hans-Martin Gauger in der FAZ: „Entweder man stellt sich auf die Seite der Sprache, oder aber das mit der ,Sichtbarmachung der Frau' ist einem dermaßen wichtig, dass man es vorzieht, sich gegen die Sprache zu stellen.“ (FAZ 10.07.2013) Damit wurde „die Sichtbarmachung der Frau“ als Problem definiert, das von außen an die Sprache herangetragen wird. Der Androzentrismus der Sprache wurde als unabänderlich hingenommen, weil , [u]nsere Welt [...] nun einmal von sehr weither durch die männliche Sicht geprägt" sei. Gauger argumentierte, das generische Maskulinum sei zwar ungerecht, jedoch ökonomisch und der deutschen Sprache (und nicht nur dieser) immanent. Die feministische Sprachkritik überschätze die bewusstseinsbildende Macht der Sprache. Kostenlose Kitaplätze seien für die Gleichstellung ungleich wichtiger als „sprachwidrige Regelungen“ (alle FAZ 10.07.2013). Damit konstruierte Gauger Sprache als etwas Vorpolitisches, das außerhalb gesellschaftlicher Entwicklungen stehe, nur immanenten Regeln folge und geringen Einfluss auf Konstruktionen von Wirklichkeit habe.

Zwei Texte begründeten die vermeintlich fehlende Verständlichkeit genderbewusster Sprache mit Zitaten, die für ein spezielles Publikum gedacht und für eine allgemeine Leser_innenschaft eher unverständlich waren. In einem Streiflicht (SZ 02.05.2014) wurde ein Zitat aus dem persönlichen Blog von Lann Hornscheidt, frühere_r Professor_in für feministische Sprachwissenschaft an der HU Berlin, angeführt. Der Blog richtet sich an Hornscheidts wissenschaftliche und aktivistische Community. Auch der Text der Fachschaft Sprachwissenschaften an der HU Berlin, über den sich die Welt (19.11.2014) lustig machte, war für ein bestimmtes und eben nicht für ein allgemeines Publikum gedacht. Die Kritik an der mangelnden Verständlichkeit trägt den Anspruch an die Gender Studies heran, wissenschaftliche Texte müssten immer auch für ein allgemeines Publikum verständlich sein, der 
überzogen ist - weil von kaum einer wissenschaftlichen Disziplin einlösbar -, und damit die Gender Studies diffamiert.

Nur in einem Text (Welt 03.05.2014) wurde anerkannt, dass wenn sich ,grundsätzliche Übereinkünfte der Gesellschaft" änderten, sich dies auch auf das Deutsche auswirken müsse. Zwar konstruierte der Artikel weiterhin einen Gegensatz zwischen Sprachästhetik und politischen Forderungen. „Doch falls sich politisch eine Mehrheit für solche Eingriffe in die Baupläne der Sprache fände, sind sie absolut vorstellbar.“(Welt 03.05.2014)

Den Texten der Frame-Gruppe Vorwurf der politischen Instrumentalisierung von Wissenschaft ist die fehlende ernsthafte Auseinandersetzung mit dem Gegenstand, den Gender Studies und der genderbewussten Sprache, gemein. Es dominieren Ironie und lächerlich machende Stilmittel. Die Artikel lassen wissenschaftliche Redlichkeit vermissen, weil mit bewussten oder unbewussten Fehlinterpretationen von Grundbegriffen aus der Geschlechterforschung hantiert wird (vgl. Näser-Lather in diesem Band). Auch an journalistischer Sorgfaltspflicht mangelt es, weil zwar Kritiker_innen der Gender Studies zu Wort kommen, Vertreter_innen der Disziplin aber nur in aus dem Zusammenhang gerissenen und für ein allgemeines Publikum teils unverständlichen Zitaten. Letzteres gilt auch für den Frame Politische Beeinflussung von Sprache.

\subsection{Frame ,Hinterfragung von Männlichkeiten“}

Der Frame Hinterfragung von Männlichkeiten war in den Jahren 2007 bis 2012 mit sechs von insgesamt zwölf Artikeln stark vertreten. Die Themen sind divers: Verunsicherung durch den Zweite-Welle-Feminismus, männliche Bildungsverlierer, grünes Männer-Manifest, Zukunftsfähigkeit von Männlichkeit, Gesundheit, Vereinbarkeit von Familie und Wissenschaft. 2013 nutzten zwei SZ-Streiflichter diesen Frame. Danach tauchte der Männlichkeiten-Frame erst 2016 wieder auf, hauptsächlich im Kontext der Islam- und Integrationsdebatte. Vier Beiträge befassten sich aus unterschiedlichen Perspektiven mit wehrhafter, körperlich durchsetzungsfähiger, verteidigungs- und gewaltbereiter Männlichkeit. Es lassen sich in diesem Frame also zwei Phasen erkennen: Zunächst wurden allgemein Geschlechterrollen und Männerrechte problematisiert. Mit der Verstärkung ethnosexistischer Diskurse (vgl. Bergold-Caldwell/Grubner in diesem Band) nach dem „Ereignis Köln“ (Dietze 2016) rückte eine vermeintliche Schutzfunktion von Männern gegenüber Frauen und der Familie in den Fokus.

Alle Artikel der ersten Phase verhandelten den Wandel von Männlichkeiten. Unterschiede gab es in der Frage, wie dieser Wandel zu bewerten sei. Die meisten Texte positionierten sich neuen Männlichkeiten gegenüber aufgeschlossen. Nach SPON (08.05.2007) seien Feminismus und Frauenbewegung zwar „nervig“ gewe- 
sen, hätten jedoch für alle Beteiligten ,neue Chancen“ eröffnet. In der SZ (11.03. 2011) lautete die Botschaft eines Artikels über die Angst von Männern vor Vorsorgeuntersuchungen, Männer müssten gesundheitsbewusster werden. In der FAZ (25.01.2012) wurden die traditionalistischen Rollenvorstellungen eines einseitig mit Väterrechtler_innen besetzten Podiums zum Thema wissenschaftliche Karriere und Vaterschaft in der Max-Planck-Gesellschaft kritisiert.

Hingegen konstruierte ein Artikel über männliche Bildungsverlierer in der Welt (24.04.2008) einen Gegensatz zwischen erfolglosen Jungen und erfolgreichen Mädchen. Ursächlich seien Rahmenbedingungen, die auf entwicklungsbiologische Eigenheiten der Jungen keine Rücksicht nähmen. In der Forderung, männliche Kinder und Jugendliche sollten durch Angebote von Männern und gemeinsame Aktivitäten mit Vätern wieder zum Lernen motiviert werden, steckt implizit die Festigung traditioneller Männlichkeit, weil nicht expliziert wurde, was genau die Jungen von den erwachsenen Männern lernen sollten. Die SZ stellte mit Verweis auf die Soziologin Hanna Rosin die Zukunft von Männlichkeit generell in Frage:

„Was, wenn die moderne, postindustrielle Gesellschaft schlicht und einfach eher auf Frauen zugeschnitten ist? [...] Was, wenn die Aufhebung der bisherigen Ungleichheit zwischen den Geschlechtern eine neue Form von Ungleichheit hervorbringen würde? [...] Was also, wenn aus der bisher unverdienten Besserstellung der Männer in Zukunft eine verdiente, weil auf besserer und legitimer Chancennutzung beruhende Besserstellung der Frauen werden würde?" (SZ 25.06.2010).

Trotz dieser sehr unterschiedlichen Bewertung des Wandels von Männlichkeit(en) machten fast alle Artikel Frauen als Verursacherinnen der Probleme aus: aufgrund von Verunsicherung und Ausschluss von Männern durch den Feminismus (SPON 08.05.2007); durch auf Mädchen zugeschnittene Bildungskonzepte und weibliches Fachpersonal in Kitas und Schulen (Welt 24.04.2008); durch die einseitige Bevorzugung von Frauen in der Gender-Forschung (SZ 11.03.2011); durch grüne Frauen, die Männer aus ihren Beratungen ausschlössen (SPON 15.04.2010).

In der zweiten Phase von Männlichkeits-Diskursen verhandelten die Beiträge eine wehrhafte, körperlich durchsetzungsfähige und gewaltbereite Männlichkeit. Zwar wollte der Kolumnist Jan Fleischhauer (SPON 30.05.2016) sein Plädoyer für mehr körperliche Durchsetzungsfähigkeit „strikt genderneutral“ verstanden wissen, legte aber durch seine Beispiele und Argumente nahe, dass es ihm um Jungen und Männer ging. Ein Welt-Autor fragte, „ob wir im Hochgefühl des Gender-Mainstreaming ,männlich“ konnotierte Tugenden wie Verteidigungsbereitschaft oder körperliche Durchsetzungsfähigkeit nicht allzu forsch entwertet haben“" (Welt 11.02.2016). Männern hierzulande sei die Selbstbehauptung abhanden gekommen. Dabei wurde Gewalt als etwas von außen Kommendes imaginiert, gegen die Män- 
ner sich und ihre Frauen verteidigen müssten. Alltägliche Gewalt, die hierzulande überwiegend von Männern ausgeht, wurde nicht thematisiert.

Der Väterrechtler Ralf Bönt (Welt 23.02.2016) drehte in einem Artikel über die Situation von Vätern unehelicher Kinder die bisher beschriebene Argumentation um. Da Väter in der Familie fehlten, hätten Jungen keine männlichen Vorbilder. Die von männlicher Leistungsfähigkeit abhängige Gesellschaft fordere Männlichkeit aber ein. Jungen versuchten deren Fehlen mit Übermännlichkeit zu kompensieren, definiert als Drogenkonsum, Suizidalität, Gewalt- und Opferbereitschaft, Fremden- und Frauenfeindlichkeit sowie gesundheitliche Vernachlässigung (Welt 23.02. 2016).

Auch in allen Texten dieser Phase wird die Verantwortung für die Probleme Frauen oder ,dem Feminismus‘ zugewiesen. Unter ihnen sei die gewaltfreie Erziehung, die Jungs verweichliche, besonders verbreitet (SPON 30.05.2016); aufgrund des Gender Mainstreaming seien männliche Tugenden verloren gegangen (Welt 11 . 02.2016). Bönt hält die ,,bessere Integration des Vaters in die innere Familie“ für eine „logische Folge der Öffnung der Arbeitswelt für Frauen und ihrer Befreiung aus der zwanghaften Mutterschaft“. Sie erlaube endlich auch ,die notwendige Neuorientierung aller Männer nach dem Verlust der Bedeutung als unentbehrliche Arbeitskräfte, welche Technik und Feminismus mit sich brachten“ (Welt 23.02.2016). Während Scheele (2016) vor allem mit Bezug auf die Diskurse um die Bildungspläne in Baden-Württemberg und Hessen einen Wandel von einem männer- zu einem familienzentrierten Antifeminismus konstatiert, zeigt sich in den untersuchten Medien eher eine diskursive Resouveränisierung von Männlichkeiten (vgl. Forster 2006). Diese bezieht sich zwar teilweise auf Familie. Ihr Schutz dient der Rechtfertigung der proklamierten verteidigungsbereiten Männlichkeit. Gleichwohl steht Männlichkeit, und eben nicht Familie, im Zentrum der Argumentation.

\subsection{Frame-Gruppe ,Wertschätzende Diskurse über geschlechtliche Vielfalt‘}

Neben dieser Mehrheit, gender'-kritischer Problematisierungen gibt es einige Texte, deren Tenor nicht die heteronormative Ordnung affirmiert, sondern die Unübersichtlichkeit der Kategorie Geschlecht wertschätzend verhandelt. Im Frame Verständnis für Einzelschicksale werden homosexuelle und Trans-Personen porträtiert. Texte über Kunst und Kultur, die die Kategorie Gender zum Thema haben, werden im Frame Spiel mit Geschlecht im Feuilleton gefasst.

Zum Frame Verständnis für Einzelschicksale gehören vier Artikel, drei auf SPON und einer in der FAZ, in denen empathisch schwule und Trans-Lebensweisen verhandelt wurden. Die FAZ (20.06.2009) porträtierte eine zunächst heterosexuelle Beziehung, in der die Frau ihr Geschlecht zum Mann angleicht. ,Wird aus 
einer heterosexuellen Liebe dann eine homosexuelle? War die Beziehung überhaupt jemals heterosexuell? Und wenn aus der Frau ein Mann wird, ist dann der Freund automatisch schwul?" (FAZ 20.06.2009) Der Text mündet in einer Art Normalisierung. Obwohl der Freund sich nicht als schwul bezeichnen würde, sei das Paar zusammengeblieben, weil es sich zusammen verändert habe, wie das bei jedem guten Paar der Fall sei. Das Paar wird damit zum Vorbild für heterosexuelle Beziehungen gemacht.

Auf SPON (21.09.2015) wurde in einer Reportage die Leidensgeschichte eines transidenten Schülers problematisiert. Eine zweite Geschichte handelte von einem schwulen Vater in Südtirol, der im Geheimen lebte und mit niemandem reden konnte (SPON 13.12.2015). Beide Features verhandelten Solidarität als Lösung: Ein schwuler Lehrer unterstützte den Trans-Jugendlichen, ein Selbsthilfeverein den homosexuellen Vater. Daneben wurde die Verfilmung des Jugendbuchbestsellers „Die Mitte der Welt" besprochen, in deren Zentrum eine schwule Liebesgeschichte steht (SPON 10.11.2016). „Dass [...] die Hauptfigur ein schwul empfindender 17-Jähriger ist, der sich im Verlauf der Handlung zum ersten Mal verliebt, ohne dass ein Coming-out in seiner Familie nötig wäre, war bei Erscheinen des Romans etwas Neues.“ (ebd.).

In der Gesamtschau fällt der androzentrische Bias der Texte auf. Berichtet wird über männliche Homosexuelle und Trans-Männer. Frauen spielen in den Geschichten Nebenrollen. Im gesamten Sample der Untersuchung gibt es keine vergleichbaren Texte über Frauen. Während traditionell Frauen der Privatsphäre zugewiesen und damit für die Beziehung zuständig gemacht wurden und Männer in der Öffentlichkeit standen und in ihrer Professionalität dargestellt wurden, ist es in diesem Sample anders. Diese Veränderung medialer Repräsentationen von Männlichkeit(en) wäre weiter zu untersuchen.

Im Frame Spiel mit Geschlecht im Feuilleton wurde in sechs Artikeln in der SZ und auf SPON Geschlecht als Gegenstand, Stilmittel oder Ausdrucksform künstlerischer Arbeiten verhandelt. Thematisiert wurde in der Besprechung einer Ausstellung des Münchener Kunstvereins die soziale Konstruiertheit von Geschlecht (SPON 19.10.2007). In der Besprechung einer CD der Sängerin Janelle Monaé wurde die Uneindeutigkeit geschlechtlicher Zuordnung in ihrem Auftritt verhandelt (SZ 17.09.2013). Im Porträt des Designers J.W. Anderson war Thema eine Art Gender Change. Anderson bediene sich für seine Modelinien bei der Garderobe des jeweils anderen Geschlechts und nenne das shared wardrobe (SZ 10.01.2015).

Drei Theater-Kritiken thematisierten die Funktion von Geschlecht für die jeweilige Inszenierung. In einer Kritik einer Inszenierung von Brechts Der gute Mensch von Sezuan wurde ein comic-haftes Spiel als Lösung des Problems von Geschlechterstereotypen verhandelt (SPON 17.04.2010). In einer Inszenierung des Stückes Jalta des Dramatikers Lucas Svensson wurden alle ausschließlich männlichen Figuren von Frauen gespielt (SZ 25.09.2013). Doch in der Kritik wurde der ,genderpoli- 
tische Sinn dieser Besetzungspointe“ in Frage gestellt. In der Rezension einer Inszenierung von Shakespeares Was ihr wollt erschien das Verwirrspiel um Geschlecht nur als Fassade. Zugleich wurde die das Stück rahmende UnterwasserKulisse als Geschlechter-Metapher interpretiert.

„Fische sind uns Menschen in Genderfragen ja weit voraus. Es gibt Arten, die ihr Geschlecht wechseln - und zwar ohne lästige Behördengänge und Bundesverfassungsgerichtsbeschlüsse. Es gibt Hermaphroditen, die gesellschaftlich voll akzeptiert sind und sich nicht jedes Mal entscheiden müssen, ob sie auf eine Männer- oder auf eine Frauentoilette gehen. Bei der Fortpflanzung sind Fische sogar klar im Vorteil: Sie haben die größere Auswahl. Manche befruchten sich selbst. Andere vermehren sich ganz ohne Geschlechtsverkehr.“ (SZ 03.03.2015)

Aus diesem Grund sei die Meeres-Kulisse gewählt worden. Resümee: Die Gegenwart der Fische sei vielleicht die Zukunft der Menschen.

\subsection{Bedrohungsszenarien in den konservativen Blättern, Differenzierung in den liberalen Medien}

Die Zuordnung der Frames zu den Medien bestätigt im Wesentlichen die Strukturierung des Samples in zwei konservative Medien (FAZ, Welt), die den ,Genderismus'-Diskurs befeuern, und zwei liberale Medien, bei denen eine differenziertere Perspektive zu erkennen ist. Dabei deckt kein Medium alle Frames ab. Den geringsten Unterschied zwischen den Medien zeigen die Frames der Gruppe Politische Instrumentalisierung von Wissenschaft. Die anti-, genderistischen` Diskurse über Gender Studies und eine genderbewusste Sprache finden offensichtlich bis in liberale Medien hinein Akzeptanz. Sie müssen daher als wichtige Pfeiler einer BrückenFunktion von Gender-Diskursen angesehen werden. Gemeinsames Merkmal ist das Fehlen einer wissenschaftlich beziehungsweise journalistisch gründlichen und redlichen Recherche und Argumentation. Die Autor_innen missachten dabei genau die Ansprüche, an denen die Gender Studies beziehungsweise genderbewusste Sprache nach ihrer Auffassung scheitern.

Ansonsten gibt es große Unterschiede zwischen den Medien. In der FAZ dominieren mit fünf Texten die ablehnend-kritischen Frames der Gruppe Angriffe auf Heteronormativität. Die meisten problematisieren eine vielfaltsorientierte Pädagogik. In einem weiteren Artikel wurden Regeln einer demokratischen Öffentlichkeit bezogen auf die Vollverschleierung formuliert. Zweiter Schwerpunkt in der FAZ sind Gender Studies als Pseudowissenschaft und genderbewusste Sprache als politische Beeinflussung. Der Frame Hinterfragung von Männlichkeiten spielt eine geringe Rolle. 
Ähnlich sieht es in der Welt aus. Hier bildet der Frame Dominanz einer Minderheit über die Mehrheit zum Thema queere Rechte und Ehe für alle mit drei Texten den Schwerpunkt. Der Frame Bedrohungs-Szenarien (zwei Texte) bezieht sich ebenfalls auf die Ehe für alle sowie auf vielfaltsorientierte Pädagogik. Als Unterwerfungs-Szenarien wurden je ein Artikel über Feminismus und über den Islam gerahmt. Unter dem mit der Gruppe Angriffe auf Heteronormativität verknüpften Frame Regeln einer demokratischen Öffentlichkeit wurden eine Selbstverständigungs-Debatte im Kontext von Integration und der Dialog zwischen queeren Menschen und der so genannten Mehrheitsgesellschaft veröffentlicht.

Ganz andere Schwerpunkte setzte die SZ. Zwar gab es auch zwei Texte im Frame Bedrohungs-Szenarien, die sich kritisch mit der vermeintlichen Bedrohung von Männern durch erfolgreiche Frauen und mit dem Konflikt um den Bildungsplan in Baden-Württemberg auseinandersetzten. Zugleich war die SZ das Medium mit den meisten Texten im Frame Hinterfragung von Männlichkeiten. Die Texte thematisierten unterschiedliche Aspekte: Islam, Gesundheit, Zukunftsfähigkeit, sowie Streiflichter zum Thema Kochen und Vergesslichkeit. Mit vier Artikeln enthielt das SZ-Sample auch die meisten Texte über das Spiel mit Geschlecht im Feuilleton. Hingegen spielten Gender Studies als Pseudowissenschaft (ein Text) und die politische Beeinflussung der Sprache (ein Text) nur eine untergeordnete Rolle.

Auch bei SPON hatten kritisch-ablehnende Frames eine geringe Bedeutung. Lediglich ein Artikel über die Entscheidung der Universität Potsdam für das generische Femininum in der Grundordnung wurde als Unterwerfung unter Feministinnen gerahmt. Die Texte über Gender Studies als Pseudowissenschaft waren differenzierter als in der FAZ und der Welt. Zwar kam in einem Text der umstrittene Entwicklungsbiologe Ulrich Kutschera ausführlich zu Wort. Eine Woche später folgte jedoch eine Replik. Nicht ganz so bedeutend wie in der SZ, aber dennoch markant waren die drei Texte im Frame Hinterfragung von Männlichkeiten. Einer befasste sich mit der Verunsicherung junger Männer angesichts der zweiten Welle der Frauenbewegung in den 1970er Jahren, ein anderer ironisierte das grüne MännerManifest. 2016 diskutierte der konservative Kolumnist Jan Fleischhauer die seiner Ansicht nach negativen Konsequenzen gewaltfreier Erziehung für die Verteidigungsbereitschaft von Männern. Bei fünf Texten handelt es sich um wertschätzende Diskurse über geschlechtliche Vielfalt: je ein empathisches Feature über das Schicksal eines schwulen und eines trans-identen Mannes, eine Film-Rezension zum gleichen Thema, eine Ausstellungs- und eine Theater-Kritik.

Insgesamt lässt sich also resümieren, dass in der FAZ und in der Welt Frames dominieren, die Gender-Diskurse als etwas Negatives und Problematisches verhandeln und die geschlechtliche und sexuelle Vielfalt insgesamt ablehnen. Abgesehen von Gender Studies und genderbewusster Sprache stehen in der SZ und auf SPON hingegen wertschätzende Diskurse zu geschlechtlicher Vielfalt und Problematisierungen des Wandels von Männlichkeit im Vordergrund. Der Unterschied lässt sich 
einerseits mit der politischen Grundausrichtung der jeweiligen Medien erklären. Andererseits lässt aber das Feature über ein zunächst heterosexuelles Paar, bei dem die Frau ihr Geschlecht zum Mann angleichen ließ, in der FAZ (20.06.2009) die Vermutung zu, dass Human-interest-Geschichten mehr redaktionelle Freiheiten eröffnen als das Nachrichten- oder Politikressort.

\section{LESER_INNEN-KOMMENTARE: ECHOKAMMERN ODER KRITISCHE REZEPTION?}

Der Untersuchung von Leser_innenbriefen in den drei Tageszeitungen und von Online-Kommentaren auf SPON lag die Frage zugrunde, wie Leser_innen die Aussagen in den Artikeln affirmieren, differenzieren oder kritisieren. In die Analyse einbezogen wurden nur Kommentare zum Text-Sample der Frame-Analyse. Von den 52 Artikeln, bei denen Frames identifiziert worden waren, wurden 19 von Leser_innen kommentiert. In der SZ zog nur einer von 13 Artikeln Leser_innenbriefe nach sich. Bei den anderen Medien hielten sich kommentierte und nicht kommentierte Artikel in etwa die Waage. Da die Schwelle online zu kommentieren erheblich niedriger ist als einen Leser_innenbrief zu schreiben, überstiegen die Kommentare auf SPON die Zuschriften um ein Vielfaches. Deswegen wurden jeweils nur die ersten 20 Online-Kommentare in die Analyse einbezogen. Online-Kommentare sind häufig spontan, erschöpfen sich in Polemik und Ironie und sind daher nicht unbedingt mit Leser_innenbriefen in gedruckten Medien vergleichbar (Ziegele 2016: 27f). Da es in der vorliegenden Untersuchung jedoch nur um eine exemplarische Analyse in einem kleinen Sample ging, wurde auf eine analytische Differenzierung zwischen Leser_innenbriefen und Online-Kommentaren verzichtet.

In einer strukturierenden qualitativen Inhaltsanalyse wurden Argumentationen und deren Muster sowie sprachliche Stilmittel untersucht. Dafür wurden die Kommentare nach Themen sortiert. Die größte Resonanz zeitigte das Thema genderbewusste Sprache mit 24 Zuschriften und 550 Online-Kommentaren. An zweiter Stelle stand das Thema vielfaltsorientierte Pädagogik mit 19 Zuschriften. An dritter Stelle folgte das Thema Gender Studies mit zwölf Zuschriften und 503 OnlineKommentaren. Über das Thema Männerrechte und Männlichkeit(en) gab es nur zwei SPON-Artikel mit insgesamt 691 Kommentaren. Gegenüber diesen Themen fielen die Themen Islam und queere Rechte mit je drei Zuschriften weit ab.

\subsection{Genderbewusste Sprache als medienübergreifender ,Aufreger}

Die Leser_innen-Argumente zu genderbewusster Sprache lassen sich grob in zwei Gruppen einteilen. Zum einen wurde sprachimmanent argumentiert, zum anderen 
wurden Intentionen und Effekte genderbewusster Sprache diskutiert. In allen Kommentaren fanden sich ähnliche Argumentationsmuster. Unterschiede gab es im Ausma $ß$ der Polemik und der inhaltlichen Fundierung der Argumente. Nur wenige Kommentare setzten sich mit den konkreten Deutungen und Argumenten des jeweiligen Artikels auseinander. In der Regel wurde der Artikel herangezogen, um grundsätzlich die eigene Meinung kund zu tun und das Anliegen genderbewusster Sprache zu diskreditieren.

Bei sprachimmanenten Argumentationen stand die vermeintliche Sprachwidrigkeit im Zentrum. So wurden „Sternchen, Schrägstriche und dergleichen“ als ,überflüssiger Firlefanz" bezeichnet, der ,unsere schöne Sprache“ verunstalte (Überflüssiger Firlefanz, FAZ 12.11.2016) oder den Lesefluss störe (Rechtlich äußerst bedenklich, FAZ 12.11.2016). Partizip-Konstruktionen wie „Studierende“ seien nicht mit dem Substantiv, also „Student“ oder „Studentin“, gleichzusetzen, da das eine einen Vorgang bezeichne, das andere den Status (Nicht einmal dumpf erahnen, FAZ 12.11.2016). Vermeintliche „Exzesse [...] unseres Sprachfeminismus“ wurden beklagt (Die Mensch/innen/heit, FAZ 03.08.2013). In vielen Kommentaren wurden genderbewusste Schreibweisen ironisch übersteigert und mit dem Vorwurf der „Political Correctness“ kritisiert: „Bundeskanzlerix, Bürgermeisterix, [...] Ministerpräsidentix und Ministerpräsidentixen, [...] Genderixen oder Gender-Nixen usw.“ (Gendergewirr, Welt 06.05.2014). Lann Hornscheidts Vorschlag der genderneutralen $\mathrm{x}$-Endung wurde mit der polemischen Forderung verknüpft, diese Schreibweise solle für alle Bereiche des öffentlichen Lebens übernommen werden, ,insbesondere für diejenigen, die uns dies möglicherweise als verbindlich vorschreiben sollen, wie seinerzeit die Schreibweise ,StudentInnen“ oder die letzte Rechtschreibreform." Der Leser setzte also die Rechtschreibreform und das Binnen-I gleich. Behauptet wurde (fälschlicherweise), in anderen Sprachen gebe es kein vergleichbares Ansinnen (Die Mensch/innen/heit, FAZ 03.08.2013, Schutz vor Gender-Gaga, Nicht einmal dumpf erahnen, FAZ 12.11.2016).

In Kommentaren zu Effekten und Intentionen genderbewusster Schreibweisen lassen sich unterschiedliche Argumentationsmuster erkennen. Das Muster ,Haben wir denn keine wichtigeren Probleme' kontrastierte die Bedeutung genderbewusster Sprache mit anderen Problemen: der Zukunft der Menschheit (Zukunft findet ohne uns statt, FAZ 12.11.2016); den Kosten, etwa für die Umbenennung der Studentenwerke in Studierendenwerke (Rechtlich äußerst bedenklich, FAZ 12.11.2016) oder für das hochbezahlte akademische Personal (spon_2318831, SPON 04.07. 2013); oder der Qualifikation von Studierenden und Absolvent_innen (Nicht einmal dumpf erahnen, FAZ 12.11.2016). Ein weiteres Muster bezog sich auf Geschlechtergerechtigkeit und Feminismus. Mehrfach wurde eine Art KronzeuginnenArgument vorgebracht. Die Schreiberin oder eine ihr bekannte Frau fühle sich nicht diskriminiert (Ich stolpere über Schrägstriche; Gerechtigkeitsfanatikern ausgeliefert; Auf Comic-Niveau, FAZ 12.11.2016). Genderbewusste Sprache habe das Ziel, 
Männer zu unterwerfen (Ronni555, SPON 04.07.2013; Machen, nicht labern, FAZ 12.11.2016). Feministinnen wurden ironisch zum militanten Feindbild ausgerufen (moysofff) und als Feminazis beschimpft (Hänschen Wurst); Feminismus wurde als „falsch verstanden“ oder ,nicht mehr zeitgemäß“ (spon_2318831) bezeichnet (alle SPON 04.07.2013).

Einige Kommentare relativierten den negativen Tenor der Artikel, indem sie bestimmten genderbewussten Sprachformen zustimmten. Doch alles habe seine Grenzen, etwa die x-Endung (Liebe MitbürgerX), der Unterstrich oder der behauptete Punktabzug bei nicht genderbewusster Sprache im Studium (Rechtlich äußerst bedenklich). Die Sprachentwicklung dürfe nicht durch normative Eingriffe gestört werden (Liebe MitbürgerX). Sprache präge das Bewusstsein, doch „erzwungene“ Änderungen seien rechtlich bedenklich (Rechtlich äußerst bedenklich) (alle FAZ 12.11.2016).

Nur wenige Kommentare wiesen die in den Artikeln geäußerte Kritik an genderbewusster Sprache zurück. Kritisiert wurde das „Mitmeinen“ im generischen Maskulinum (Mitgemeint, FAZ 12.11.2016). Genderbewusste Sprache könne auch zum Nachdenken über „unbedachte Sprachgewohnheiten“ anregen (Thomas Mank, SPON 04.07.2013).

Argumentationsgang, Tenor und die polemischen und ironischen Stilmittel der Kommentare zu genderbewusstem Sprachgebrauch weisen in allen Medien große Ähnlichkeiten auf. Dies stützt die These, dass dieses Thema einer der inhaltlichen Pfeiler einer Brücke zwischen rechten und antifeministischen Kreisen einerseits und konservativen bis liberalen Bevölkerungsgruppen andererseits ist.

\subsection{Vielfaltsorientierte Pädagogik als Bedrohungsszenario für christlich-konservative Leser_innen}

Im Frame-Sample zu vielfaltsorientierter Pädagogik gab es fünf Artikel, drei in der FAZ und je einer in der SZ und der Welt. Leser_innenreaktionen riefen zwei Texte in der FAZ (sieben bzw. vier Briefe) und einer in der Welt (vier Briefe) hervor. Bis auf einen Leser, der argumentierte, Kinder sollten über täglich zu beobachtende Phänomene aufgeklärt werden (Unwissenheit hat noch niemandem geholfen, FAZ 30.10.2014), affirmierten alle den ablehnenden und diffamierenden Tenor der Artikel. Mit teils ähnlichen Formulierungen wurden die gängigen Argumente der Gegner_innen vielfaltsorientierter Pädagogik vorgebracht (Scheele 2016; Schmincke 2015). Dominantes Stilmittel war ein besorgter bis bedrohlicher Tenor.

Ein Argumentationsmuster war der Kontrast zwischen der heilen Welt der Kleinfamilie und behaupteten Intentionen vielfaltsorientierter Pädagogik im GutBöse-Schema. Der behütenden traditionellen Familie wurden Kinder aus ,zerrissenen Familien“ oder in die Fremdbetreuung abgeschoben gegenübergestellt. Solche 
Kinder wünschten sich nichts sehnlicher als eine intakte traditionelle Familie, welche die vielfaltsorientierte Pädagogik zum Auslaufmodell erklärt habe (Wer fragt eigentlich die Kinder, FAZ 27.07.2011). Der „Verstaatlichung“ der Kindererziehung in Betreuungseinrichtungen und Untergrabung der elterlichen Autorität (Sophia Kuby zu „Kinderliebe, Elternliebe“, Welt 29.10.2007) wurde „Werteerziehung“" als ausschließlich den Eltern obliegend entgegengesetzt (Neue Aufklärung, FAZ 27.10.2014). Stabile Beziehungen und Liebe in Ehe und Familie wurde mit Sex als Konsumgut oder Selbstverwirklichung sowie massenhafter „Tötung von ungeborenen Kindern im Mutterleib“ kontrastiert (Welche Last wird Kindern noch aufgebürdet?, FAZ 28.07.2011; Samuel Beckmann zu „Kinderliebe, Elternliebe“, Welt 29.10.2007). Das unschuldige Kind als Chiffre (Schmincke 2015) wurde imaginiert als von geschlechtlicher Vielfalt „erschlagen“ (Welche Last wird Kindern noch aufgebürdet?, FAZ 28.07.2011), als „missbraucht“ durch vielfaltsorientierte Pädagogik (Missbrauch, FAZ 27.10.2014), als Kind, das eigentlich nur mit Puppen oder Lego spielen wolle, aber zu früh mit Sexualität konfrontiert werde (Wie lange dürfen unsere Kinder noch Kinder sein?, FAZ 26.07.2011).

Ein weiteres Argumentationsmuster bestand in der verschwörungstheoretischen Kombination der Vorwürfe Ideologie, Umerziehung und beschnittene Meinungsfreiheit. Mit dem Ziel der Umerziehung der Kinder (bzw. der gesamten Gesellschaft) (Nieder mit der Gender-Ideologie, FAZ 30.10.2014) verbreiteten Lobbygruppen und „sexuelle Interessenverbände“ (Wie lange dürfen unsere Kinder noch Kinder sein?, FAZ 26.07.2011) die Gender-Ideologie ,auf schleichenden Sohlen“ (Nieder mit der Gender-Ideologie; Die Hintertür-Methoden der Gender-Ideologen, beide FAZ 30.07.2011). Der Staat, das „UN-Establishment“ (Irrsinn, FAZ 27.10. 2014) und die Bundeszentrale für gesundheitliche Aufklärung (Samuel Beckmann zu „Kinderliebe, Elternliebe“, Welt 29.10.2007) betrieben dieses Geschäft ebenfalls. Hingegen würden Proteste, wie die Online-Petition gegen den Bildungsplan Baden-Württemberg, die Demos der Besorgten Eltern und die Märsche für das Leben von den Medien ignoriert (Selbsternannte Aufklärer unserer Kinder, FAZ 27. 10.2014).

Wegen der starken Übereinstimmung der Argumentationsmuster wurden im Internet die Namen der Schreibenden recherchiert. Sechs der zehn Schreibenden zum Artikel „Anleitung oder Aufklärung zum Sex“ (FAZ, 23.10.2014) über eine vielfaltsorientierte Pädagogik bewegen sich in einem christlich-konservativem oder evangelikalem Kontext (z.B. Evangelische Allianz Deutschland, freie christliche Schule). Eine Schreiberin trat als Rednerin bei einer Demo für alle auf. Sie gehören mithin dem gleichen ideologischen Umfeld an wie der Autor des Artikels. Auch einige Leser_innenbriefe $\mathrm{zu}$ anderen Artikeln lassen sich einem christlich-fundamentalistischen Umfeld zuordnen.

Zum Artikel „Kinderliebe, Elternliebe“ des Soziologen Gerhard Amendt (Welt 26.10.2007) äußerte sich Sophia Kuby, die Tochter der ultrakonservativen katholi- 
schen Publizistin Gabriele Kuby. Diese hatte dafür gesorgt, dass ein Aufklärungsratgeber der Bundeszentrale für gesundheitliche Aufklärung von Bundesfamilienministerin Ursula von der Leyen zurückgezogen wurde (Lang/Peters 2018: 13f). Die Kritik an dem Ratgeber stand im Zentrum von Amendts Artikel (Welt 26.10. 2007). Dies wiederum lobte die Tochter der Initiatorin dieser Aktion in ihrem Leserinnenbrief.

Diese Ansammlung von Zuschriften mit gleichem Tenor macht eine Absprache naheliegend. Das gemeinsame ideologische Dach ist klar erkennbar. Abgesehen davon gab es kaum Leser_innen-Reaktionen. Das lässt die Vermutung zu, dass vielfaltsorientierte Pädagogik nur für ein christlich-konservatives und fundamentalistisches Spektrum ein Problem ist, nicht jedoch für die eher konservativen Leser_innen der FAZ und der Welt insgesamt. Allerdings müsste dies mit weiteren Untersuchungen überprüft werden.

\subsection{Gender Studies: \\ Kontroverse Debatten in der Leser_innenschaft}

Zu vier der sechs Artikel über Gender Studies gab es Leser_innen-Reaktionen, zwei zu einem FAZ-Artikel, darunter ein Text einer achten Klasse zum Thema Vielfalt geschlechtlicher Identitäten (FAZ 22.06.2017), 283 beziehungsweise 200 Kommentare zu zwei SPON-Artikeln und zehn Leser_innenbriefe zu einem SZ-Artikel. Die Resonanz war erheblich vielfältiger als beim Thema genderbewusste Sprache.

Ein wesentlicher Diskursstrang auf SPON und in der SZ bezog sich auf die biologische oder soziale Bedingtheit von Geschlecht. Mit der Behauptung einer der Kultur vorgängigen Natur wurden traditionelle Vorstellungen von Geschlecht affirmiert. Angeführt wurde ein vermeintlich sozialisationsunabhängiges typisches Verhalten von Jungen und Mädchen (weltgedanke), die negativen Folgen erzwungener geschlechtsuntypischer Rollen (keksguru), die Sinnhaftigkeit traditioneller Rollenverteilung, die den Wünschen der meisten Männer entspreche (Paul Panda). Behauptet wurde, die Hirnforschung würde immer mehr Unterschiede zwischen Männern und Frauen entdecken (stevegee) (alle SPON 04.09.2015).

Einige Kommentare auf SPON, die die Kritik an den Gender Studies zurückwiesen oder relativierten, bezogen sich ebenfalls auf das Verhältnis von Natur und Kultur (Kiesch), lobten die eigene gleichberechtigte Beziehung (rudisresterampe, Ich bin Froh [sic!], dass meine Frau...,) und empfahlen dem Evolutionsbiologen Kutschera, in ein arabisches Land mit seinem untertänigen Frauenbild auszuwandern (epikur) (alle SPON 04.09.2015).

Der Ideologie-Vorwurf wurde mit der vermeintlichen Ignoranz der Gender Studies gegenüber der biologischen Bedingtheit von Geschlecht verknüpft. Es fielen Begriffe wie „Gender-Wahnsinn“, Gender als Angriff auf die „gesunde Identität des 
Menschen“ (stevegee) „Ideologen und Sekten“, „linke Volksbeglücker“ und „Sprachpolizist“ (oldeagle), „Schaumschlagen“ (uvendrix) (alle SPON 04.09.2015) oder „Wünschelrutengänger“ (Nomen Nescio, SPON 12.09.2015).

Mit dem Ideologie-Vorwurf korrespondierte auch der Vorwurf der ,Meinungsdiktatur“. „[P]olitische Korrektheit“" (bei der Sprache) werde selektiv erzwungen, etwa durch die Drohung, studentische Arbeiten andernfalls abzuwerten (pock, Provokant?). Eine Stellungnahme der Universität Kassel zu Kutscheras Einlassungen wurde als „Abmahnung“ bezeichnet und als Beleg dafür angeführt, dass Abweichungen von der ,reinen Lehre“ der Gender-Forschung abgestraft werde (scr_berlin) (beide SPON 04.09.2015). In der FAZ äußerte ein Leserbrief-Schreiber, er als ehemaliger DDR-Bürger müsse nun feststellen, dass bei ,bestimmten Themen“ in der öffentlichen Debatte Meinungsfreiheit fehle. Indirekt verglich er die Gender Studies mit Zensur in der DDR (Meinungsfreiheit, FAZ 06.06.2015).

In einem dritten Diskursstrang wurde den Gender Studies die Wissenschaftlichkeit abgesprochen. Die „evidenzbasierten“ Naturwissenschaften wurden von einem behaupteten Wissenschaftsbegriff der Gender Studies abgegrenzt, die in alle anderen Disziplinen hineinregieren wollten (christian_gapp) und die wissenschaftlichen Prinzipien von Falsifizierbarkeit und Reproduzierbarkeit ignorierten (Nomen Nescio). Daneben wurde ein Gegensatz zwischen (Frauen-)Politik und Wissenschaft konstruiert (Criticz) (alle SPON 12.09.2015).

Die Leser_innenbriefe zum SZ-Artikel „Krampfzone“ (16.04.2016) hoben sich von diesen Argumentationsmustern deutlich ab. Der Natur-Kultur-Diskurs wurde mit dem Hinweis auf den Konstruktivismus als Basis der meisten Geistes- und Sozialwissenschaften aufgegriffen. Wer dem Konstruktivismus die Wissenschaftlichkeit abspreche, greife das Wissenschaftssystem insgesamt an (Basisliteratur vieler Fächer) und wolle soziale Phänomene mit den Methoden der Naturwissenschaft analysieren (Sozial- nicht Naturwissenschaft). Vorgeworfen wurden dem Autor „Unterstellungen [...] aus völliger Unkenntnis ihrer Grundannahmen“ (In den kulturellen Kontext setzen), „Steinzeit-Holzhammer“, „,wild zusammengewürfelte(n) Studien“ und „fragwürdige Thesen“ (Ist das Ihr Ernst?). Er habe seine journalistische Sorgfaltspflicht missachtet (Basisliteratur vieler Fächer) (alle SZ 21.04.2016). Dass sich unter den Leser_innenbrief-Schreiber_innen auch namhafte Geschlechterforscher_innen befanden, ist an sich nicht verwunderlich, da die SZ ein liberales intellektuelles Publikum anspricht. Allerdings ist bemerkenswert, dass - anders als nach entsprechenden Artikeln in der FAZ und der Welt - sich keine ,Gender'Kritikerinnen äußerten. Das konterkariert die Befunde der Frame-Analyse, wonach Kritik an den Gender Studies zu den Pfeilern einer Brücke zwischen rechten und antifeministischen Kreisen einerseits und manchen bürgerlichen Gruppen andererseits gehören könnte.

Wie bei anderen Topoi der, Genderismus'-Debatte auch, sind viele Kommentare in ironischem und polemischem Ton gehalten. Bei den Online-Kommentaren zu 
dem Artikel „Die gekränkten Kritiker“ (SPON 12.09.2015) kam ein weiteres Stilmittel hinzu. Der Autorin wurde die Kompetenz abgesprochen, sich überhaupt zu diesem Thema zu äußern. Sie habe die theoretischen Grundlagen der Gender-Forschung nicht richtig wiedergegeben (im_ernst_56); sie verfolge einen „falsche[n] Denkansatz", ziehe sachliche Kritik auf die persönliche Ebene und führe kein sachliches Argument an (Edelstoffl). Einige Kommentator_innen maßten sich ein Urteilsvermögen an, das sie der Autorin absprachen: clausbremen erklärte apodiktisch: „Sie meint tatächlich [sic!], Gender-Forchung [sic!] sei eine ,Wissenschaft“ Ich sage: NEIN, ist sie nicht“" (alle SPON 12.09.2015).

\subsection{Männlichkeiten: Affirmation der männlichen Beschützerrolle}

Zum Thema Männlichkeiten wurden zwei Artikel ausschließlich auf SPON kommentiert. Im ersten Beitrag „Abschied vom Macho-Mann“(SPON 15.04.2010) ging es um ein Männer-Manifest der Grünen (450 Kommentare). Im zweiten Beitrag „Aua Papa“ (SPON 30.05.2016) kontextualisierte Jan Fleischhauer in seiner Kolumne gewaltfreie Erziehung und sinkende Verteidigungsbereitschaft (241 Kommentare) (vgl. Abschnitt 4.4).

Die Kommentare des ersten Artikels wurden durch die einleitende Frage, wo die Grünen heute stünden, vorstrukturiert. (Wo stehen die Grünen heute, SPON 11.01. $2010^{13}$ ). Aufgrund dessen tauchte das Thema Wandel von Männlichkeiten nicht auf. Die Kommentare zu Fleischhauers Kolumne kombinierten das Thema gewaltfreie Erziehung und Männlichkeit in ähnlicher Weise wie der Artikel selbst. Gewaltfreie Erziehung erziehe zur Wehrlosigkeit. Vor allem Jungs müssten sich schon im Sandkasten zu wehren wissen (jojack). Da seien Männer den Frauen körperlich voraus, meinte ein Kommentator (Curiosus). Diese Auffassung sei heute jedoch in der Minderheit (stammtischschreck). Die Verantwortung für die Misere wurde Frauen zugeschrieben. Die ,sorry, zumeist weiblichen“ Vertreter gewaltfreier Erziehung müssten anerkennen, dass auch verbale Gewalt, die im Kindergarten vor allem von Mädchen gegen Jungen ausgeübt werde, Gewalt sei (dr.joe66). Daneben wurden „desorientierte Mütter“ für vermeintlich verweichlichte Jungs verantwortlich gemacht. Damit sei das Ziel der Gender-Diskussion erreicht, Unterschiede zwischen den Geschlechtern zu nivellieren (Tiananmen).

Großen Raum nahm die Frage der Legitimität von Gewalt ein. Gewalt dürfe nicht das erste Mittel sein, man müsse sich aber auch nicht zum Opfer machen lassen (Leser 161). Doch wer seine Familie verteidige, laufe Gefahr, vor Gericht als

13 Der zugrunde liegende Artikel „Abschied vom Macho-Mann“ wurde am 15.04.2010 veröffentlicht. Allerdings tragen die in die Untersuchung einbezogenen Kommentare (möglicherweise durch einen technischen Fehler der Website) alle das Datum 11.01.2010. 
Aggressor hingestellt zu werden, während Täter zum Opfer gemacht würden (Suppenkoch). Weitere Aspekte waren die fehlende Bereitschaft zum Eingreifen als Konsequenz zunehmender Gewaltbereitschaft der Gewalttäter, als „Bammel“ oder Angst davor, sich die Hose schmutzig zu machen (huger56; tuedelich). Es gebe „Bevölkerungsschichten“, in denen es ,halt eben rustikaler“ zugehe. Mit dem Hinweis, es sei „einfach nützlich wenn einem Hemmschwelle [sic!] für eine rustikale Gegenantwort nicht unendlich hoch anerzogen wurde“, wurde die Brücke zum Thema gewaltfreie Erziehung geschlagen (licht2009).

Zwei Kommentatoren schilderten das eigene körperliche Eingreifen im Tenor demonstrativer männlicher Dominanz und Selbstzufriedenheit. Der eine beschrieb detailliert, wie er eine Frau vor den Übergriffen ihres Begleiters geschützt habe. Die direkte Ansprache mit dem Satz „Ich sage Ihnen, was ich getan habe“ und Formulierungen wie „freundlich bestimmt“ und „ganz schnell wieder brav“ suggerieren Selbstsicherheit. Dies mündete in der Feststellung, „ein selbstsicheres Auftreten und der Mumm im Zweifelsfall auch ,eine“ zu kassieren“ hätten gereicht (sponfacebook-10000361426). Ein anderer beschrieb, wie er als Junge den kleinen Bruder drangsaliert und trotzdem den Anschein gewaltfreier Erziehung gewahrt habe (murksdoc).

Einige Kommentare modifizierten oder relativierten Fleischhauers Aussagen. Ein Kommentator, der sich als Pazifist bezeichnete, unterschied zwischen Gewalt und Schwäche (Unbekanntgeblieben); ein anderer differenzierte zwischen einem Konflikt, den man mit gewaltfreier Kommunikation nach Rosenberg lösen könne, und einem Überfall wie in Köln. Solle Kindern Selbstverteidigung für solche Fälle beigebracht werden, „müssen wir sie regelmäßig von fremden Menschen grundlos überfallen lassen und ihre Reaktion darauf trainieren“ (masselquuu). Drei Kommentare wiesen Fleischhauers Aussagen explizit zurück. Sie differenzierten zwischen Gewalt im Kinderzimmer und Selbstverteidigung (Gretchen2.0; tuedelich) und fragten, ob Fleischhauer seine Überlegungen selbst praktizieren und seinen Sohn ohrfeigen würde (TLB) (alle SPON 30.05.2016).

In den ersten 20 Kommentaren wurde dreimal Bezug zum „Ereignis Köln“ (Dietze) genommen. Ansonsten blieb Gewalt überwiegend ein von außen kommendes Abstraktum. Das Konkreteste waren die Rede von Bevölkerungsschichten, in denen es ,rustikaler' zugehe, sowie die Schilderungen persönlichen Eingreifens. Überwiegend wurde Gewalt Anderen zugeordnet oder als von außen hereinbrechend konstruiert. Alltägliche Gewalt in der Gesellschaft, etwa geschlechtsbasierte und sexualisierte Übergriffe, Gewalt gegen Kinder und Kindesmissbrauch wurden nicht angesprochen. Gewalt wurde also externalisiert. Die Kommentator_innen schrieben Männern die Rolle als Beschützer zu. Darauf müssten sie von Kindesbeinen vorbereitet werden. Daran, dass das heute nicht mehr der Fall sei, trügen auch Frauen die Schuld. 


\subsection{Queere Rechte: Sind Leser_innen konservativer Blätter aufgeschlossener als ihre Zeitung?}

Das Sample zu queeren Rechten für die Frame-Analyse bestand aus neun Artikeln. Nur zwei Artikel in der Welt zogen Leser_innenbriefe nach sich. In beiden wurde mit unterschiedlichen Facetten eine homosexuelle Avantgarde imaginiert, die die Macht über die ,normale“ Bevölkerungsmehrheit übernehmen wolle. Dazu wurde auch das Beispiel des vielfaltsorientieren Bildungsplans in Baden-Württemberg herangezogen. Die insgesamt drei Leserbriefe wiesen die polemische Argumentation der Artikel zurück. Ein Leserbrief führte eigene Erfahrungen mit homosexuellen Personen im Bekanntenkreis an (Keine Hybris, Welt 17.06.2013). Der andere warnte vor rechten Vereinnahmungen und erklärte Regenbogenfamilien zu normalen Lebensentwürfen (Mehr Gelassenheit, Welt 17.06.2013). Der dritte Leser_innenbrief wies im Kontext des Bildungsplans in Baden-Württemberg den Eindruck zurück, ,dass das nach staatlichen Richtlinien erstellte Hintergrundmaterial für Lehrkräfte quasi eins zu eins in Kinderseelen transportiert wird“. Dies sei vielmehr die pädagogische Aufgabe von Lehrer_innen (Kinderseelen, Welt 04.07.2015). Angesichts der rechtskonservativen Tendenz der Zeitung Die Welt ist es ungewöhnlich, dass drei Leser_innenbriefe eine aufgeschlossene Haltung zu queeren Rechten und vielfaltsorientierter Pädagogik zeigten, ohne dass dem eine andere Meinung entgegengesetzt wurde.

\subsection{Islam: ablehnende, jedoch geringe Resonanz}

Dem Thema Islam waren im Frame-Sample acht Artikel aus den konservativen Medien zuzuordnen. Drei Artikel zogen je einen Leser_innenbrief nach sich. In einem Essay in der Welt (28.12.2015) wurde angesichts der stark gestiegenen $\mathrm{Zu}$ wanderung eine notwendige Selbstverständigungsdebatte in der Gesellschaft gefordert (vgl. Abschnitt 4.2). Der Leserbrief griff dies jedoch nicht auf. Vielmehr tat er seinen Unmut über Immigrant_innen kund, die gering qualifizierten Deutschen den Arbeitsplatz wegnähmen, weil sie mächtige Lobbys hätten (Lautstarke Lobby, Welt 30.12.2015). Auf einen Bericht über einen Gesetzentwurf zum Verbot sexistischer Werbung (Welt 12.04.2016) folgte ein Leserbrief, der polemisch die Aussage des Artikels verstärkte. Demnächst würde Frauen das Lachen oder Tragen leichter Sommerkleider verboten, der Schleier und eine Halal-Fleischquote eingeführt. Wie im Artikel wurde sexistische Werbung mit Erotik und Freiheit gleichgesetzt und insinuiert, Frauen solle eine weibliche Erscheinung verboten werden (Abschaffen, Welt 13.04.2016) (vgl. Abschnitt 4.1). In einem Gastbeitrag für die FAZ (18.08. 2016) redete der CDU-Politiker Jens Spahn einem Verschleierungsverbot in der Öffentlichkeit das Wort. Eine Leserin affirmierte dies mit der Aussage: ,[u]nsere To- 
leranz sollte da ihre Grenze haben, auch ansagen [sic!], wo Intolerante uns das Maß unserer Toleranz aufzwingen“ (Die Grenze der Toleranz, FAZ 23.08.2016) (vgl. Abschnitt 4.2).

\subsection{Themenübergreifende Varianzen: Kritik an den Gender Studies, Akzeptanz geschlechtlicher Vielfalt}

Einige Argumentationsmuster in den Leser_innen-Reaktionen tauchten themenunabhängig immer wieder auf: die Familie als Auslaufmodell, die Bedrohung der Normalität aus Mann, Frau und Kind durch eine Minderheit, die Zerstörung der Gesellschaft, Bevormundung, Gender oder Gender Mainstreaming als Ideologie, deren Grundlagen und schädliche Wirkungen regierungsamtliche Stellen vor der Bevölkerung verschwiegen. Diese Übereinstimmungen sind Hinweise auf einen übergreifenden ,Genderismus'-Diskurs, bei dem die konservativen Medien häufiger den Tenor ihrer Leser_innenschaft trafen. Bei den liberalen Medien war die Resonanz differenzierter. Während sich Leser_innen den Polemiken und Kritiken an genderbewusster Sprache und den Gender Studies überwiegend anschlossen, war die Meinungsvielfalt bei den Themen Männlichkeiten und queere Rechte selbst in konservativen Medien größer. Dies kann als Indiz dafür gelten, dass geschlechtliche und sexuelle Vielfalt bis weit in bürgerliche Kreise akzeptiert wird und kritische Diskurse nur eine begrenzte Reichweite entfalten. Auch vielfaltsorientierte Pädagogik scheint hauptsächlich für christlich-konservative und fundamentalistische Gruppen ein Aufregerthema zu sein.

\section{WIDERSPRÜCHLICHE DISKURSE: ANTIFEMINISTISCHE BRÜCKEN UND LIBERALISIERTE ABGRENZUNGEN}

Die im Forschungsstand als diskursprägend genannten Veröffentlichungen (FAZ 19.06.2006, Junge Freiheit 07.07.2006, Der Spiegel 1/2007) haben keine ausgeprägte mediale ,Genderismus'-Debatte ausgelöst. Zumindest die FAZ war spätestens seit der UN-Bevölkerungskonferenz 1994 in diese Debatte involviert. Und ein quantitativer Anstieg entsprechender Artikel ist erst ab 2011 bis 2013 parallel zum Aufstieg der AfD erkennbar. Da dieser Anstieg jedoch nichts mit der Berichterstattung über die AfD zu tun hat, ist ein tieferliegender gesellschaftlicher Trend in Form rechter und antifeministischer Mobilisierungen zu vermuten.

Auf der Ebene der Begriffe stehen Gender Mainstreaming sowie Komposita rund um die Gender-Forschung im Zentrum. Es wurden jedoch im gesamten Unter- 
suchungszeitraum von 2006 bis 2016 nur wenige externe Anlässe identifiziert, um die herum sich medienübergreifende Diskurse entfalteten. Das lässt die These zu, dass Medien im gesellschaftlichen Diskurs um Gender als Akteur_innen weitgehend eigenständig agieren. Dabei treffen konservative Medien eher als liberale die Meinungen ihrer Leser_innenschaft und dienen daher eher als Echokammern entsprechender gesellschaftlicher Gruppen. Möglicherweise handelte es sich bei den Ausgangstexten 2006/2007 um Tabubrüche, bei denen es anderen Medien zunächst nicht opportun erschien, in die Debatte einzusteigen. Die Zunahme entsprechender Thematisierungen ab etwa 2011 bis 2013 könnte hingegen als Normalisierung von medialen Gender-Diskursen begriffen werden, die durch eine Intensivierung rechter Mobilisierungen befeuert worden sein könnte.

Die Befunde meiner Untersuchung , Genderismus ' in der medialen Debatte diskutiere ich im Folgenden in fünf Thesen.

\section{1 ,Reine“ Wissenschaft und Sprache versus politische Einflussnahme (These 1)}

Bei Artikeln über und Kommentaren zu Gender Studies und genderbewusster Sprache gibt es die geringsten Unterschiede zwischen den konservativen und liberalen Medien im Sample. Diese Themen können medienübergreifend als zentrale inhaltliche Pfeiler einer diskursiven Brücke zwischen rechtsextremen, religiös-fundamentalistischen und antifeministischen Gruppen einerseits sowie gesellschaftspolitisch konservativen bis liberalen Kreisen andererseits eingeordnet werden. Der Tenor ist fast einhellig kritisch, polemisch und diffamierend. Die Texte sind geprägt von einem positivistischen und objektivistischen Wissenschaftsbegriff und einer biologistischen Perspektive auf Geschlecht. Auf dieser Basis wird ein Gegensatz konstruiert zwischen der ,reinen', unpolitischen Wissenschaft und Sprache einerseits und einer außerhalb dieser Sphären liegenden politischen Intervention von vermeintlichen (feministischen) Lobbys andererseits, die mit diktatorischen Mitteln versuchten, ihre Macht zu vergrößern. Diese Argumentationsfigur wird in den meisten Leser_innen-Kommentaren affirmiert. Das Hohelied der exakten evidenzbasierten Wissenschaft steht jedoch in frappierendem Widerspruch zur fehlenden Sorgfalt bei der eigenen wissenschaftlichen und journalistischen Argumentation der Kritiker_innen. Ein Grund dafür könnte sein, dass die Gender Studies und eine genderbewusste Sprache in solchem Ausmaß abgewertet wird, dass eine ernsthafte Auseinandersetzung unnötig erscheint. Näser-Lather weist daneben darauf hin, dass einige wissenschaftliche ,Gender'-Kritiker_innen, die in den Medien zu Wort kommen, sich damit außerhalb ihrer eigenen wissenschaftlichen Disziplin bewegen, also schlicht wenig Ahnung vom Gegenstand der Geschlechterforschung, der Kategorie Geschlecht, haben (vgl. Näser-Lather in diesem Band). 


\subsection{Liberalisierung von Geschlechterordnungen bis in konservative Kreise (These 2)}

Bei den Themen vielfaltsorientierte Pädagogik und queere Rechte deutet sich eine Liberalisierung von Geschlechterordnungen an. Zwar greifen Autor_innen in der FAZ und der Welt vielfaltsorientierte Reformen schulischer Bildungspläne und Initiativen für die Ehe für alle an. Im Zentrum der Kritik stehen die Chiffren des unschuldigen Kindes und der heilen Familie (vgl. Krüger-Kirn/Tichy und Oldemeier/ Backöfer/Maurer/Aleksin in diesem Band). In Artikeln im Kontext von queeren Rechten wird eine sexuell freizügige Avantgarde imaginiert, die die heterosexuelle Normalität zerstören wolle. Doch Beifall finden solche Artikulationen nur in den Leser innen-Kreisen, die dem Umfeld der Demo für alle zuzurechnen sind. In liberalen Medien wird die Vielfalt von Geschlecht und Sexualität hingegen als unspektakuläre neue Normalität gewertet. Diffamierende Artikel zu queeren Rechten werden selbst in der rechtskonservativen Tageszeitung Die Welt von Leser_innen zurückgewiesen. Anhaltspunkte für die diesem Befund zugrundeliegende Liberalisierung finden sich auch in der REVERSE-Fallstudie zur Ehe für alle (vgl. Fritzsche/Lang in diesem Band). Einstellungsuntersuchungen auf Basis von Daten aus dem European Social Survey deuten ebenfalls darauf hin, dass in Westeuropa nur noch unter zehn Prozent der Befragten, darunter überproportional viele sehr religiöse Menschen, Homosexualität stark ablehnen (Spierings 2018: 185f).

\subsection{Männlichkeit bleibt zentraler Topos von Antifeminismus und Anti-,Genderismus' (These 3)}

Der Frame Hinterfragung von Männlichkeit(en) hat das Sample in den ersten Jahren des Untersuchungszeitraums von 2007 bis 2012 dominiert. Die zentrale Aussage der meisten Artikel war, Männer und vor allem Jungen seien das eigentlich benachteiligte Geschlecht. Frauen im Allgemeinen und Feministinnen im Besonderen trügen die Schuld an dieser Misere. Diese Viktimisierung war und ist ein Diskursmittel antifeministischer Mobilisierung (u.a. Aigner 2012; Gesterkamp 2010; Rosenbrock 2012; Schutzbach/Lanwer 2017). Zugleich wurden damit soziale Fragen dethematisiert, wenn etwa Jungen pauschal als Bildungsverlierer bezeichnet wurden, ohne zu fragen: Welche Jungen aus welchen sozialen Milieus sind hiervon betroffen (Rieske 2011)? Insofern bestätigt sich Scheeles These von der Männerzentrierung als Ausgangspunkt antifeministischer Diskurse (Scheele 2016). Nach einem zwischenzeitlichen Abflauen tauchte der Frame in den untersuchten Medien 2016 mit Diskursen über eine verteidigungs- oder gewaltbereite, wehrhafte Männlichkeit gegen Gewalt von außen im Kontext des „Ereignisses Köln“ (Dietze) wieder auf. Der diskursiven Viktimisierung folgte also der Versuch einer Resouveränisierung, 
die auf der Stilisierung körperlich durchsetzungsfähiger Männlichkeit als Beschützerin ,unserer“ Frauen, Kinder und Familien beruhte. Gewalt wurde von außen kommenden Anderen zugeschrieben, die in die Mehrheitsgesellschaft eindringen. Im Zentrum dieses Schutzdiskurses standen in den untersuchten Medien nicht Frauen oder Familien als zu schützende Objekte, sondern Männer als schützende Subjekte. Zwar galt dieser Schutz auch der Familie. Doch Hinweise auf einen Wandel von einem männer- zu einem familienzentrierten Antifeminismus, den Scheele mit Bezug auf Angriffe auf vielfaltsorientierte Pädagogik mit den Chiffren des unschuldigen Kindes und der heilen Familie beschreibt, lassen sich zumindest im vorliegenden Sample nicht finden. Vielmehr hat sich gezeigt, dass Angriffe auf sexuelle Bildung und queere Rechte gesellschaftlich eine sehr begrenzte Reichweite haben (siehe These 2). Insofern muss Scheeles These differenziert werden. Zwar gehört die ,heile Familie' zu den rhetorischen Figuren des Schutzdiskurses. Jedoch steht sie nicht im Zentrum. Vielmehr zielt dieser auf eine Resouveränisierung traditioneller Männlichkeiten. Der Gewalt-Topos dient zusätzlich der Veranderung von Gewalttätern und damit der Dethematisierung von Gewalt durch herkunftsdeutsche Männer.

\subsection{Vereinnahmung von Gleichstellung und Feminismus für kulturalisierende Ausschlüsse (These 4)}

Obwohl das „Ereignis Köln“ (Dietze) in den Untersuchungszeitraum fiel, wurde in nur wenigen Artikeln ausschließlich in den konservativen Zeitungen die Integrations- und Islam-Debatte mit Gender-Fragen kontextualisiert. Doch in diesen wenigen Texten ist die Verknüpfung eindeutig. Zum einen werden Feminismus und Islamismus in einen Topf geworfen und als autoritär oder diktatorisch gekennzeichnet. Beide politischen Bewegungen hätten zum Ziel, ,unsere‘ Freiheit einzuschränken oder abzuschaffen, wobei unter Freiheit (bzw. Erotik) auch die sexuelle Objektivierung von Frauen, beispielsweise durch sexistische Werbung, gefasst wird. Zum anderen werden Regeln ,unserer' demokratischen Öffentlichkeit mit der vermeintlichen Rückständigkeit der Herkunftskultur von Eingewanderten kontrastiert und am Beispiel von Frauenrechten, etwa dem Recht sich unverschleiert in der Öffentlichkeit zu bewegen, illustriert. Dies schließt an Bergold-Caldwells und Grubners Analyse von argumentativen Mustern in diesem Band an, welche die behauptete erreichte Gleichheit von Männern und Frauen hierzulande mit der vermeintlichen Rückständigkeit der Herkunftskulturen Zugewanderter kontrastieren. 


\subsection{Geschlechtliche Vielfalt in medialen ,Labors‘ (These 5)}

Wertschätzende Bezugnahmen auf geschlechtliche und sexuelle Vielfalt fanden sich vor allem in Human-interest-Geschichten und im Feuilleton, jedoch fast ausschließlich in den beiden liberalen Medien. Das könnte zum einen an der jeweiligen politischen Ausrichtung liegen. Es könnte aber auch darauf hinweisen, dass das Feuilleton und Human-interest-Ressorts, wie Kunst und Kultur insgesamt, als ein Labor fungieren, in dem eine Vielfalt ausprobiert werden kann, die andernorts (noch) nicht möglich ist. Die redaktionellen Freiheiten könnten hier größer sein als im Nachrichten- oder Politikressort, wo die zentralen gesellschaftlichen Diskurse schärfer ausgetragen werden. Diese Interpretation ist vor allem vor dem Hintergrund einer Reportage über ein zunächst heterosexuelles Paar, bei dem die Frau ihr Geschlecht zum Mann angleichen ließ, in der FAZ (20.06.2009) plausibel.

Allerdings haben zumindest die Human-interest-Geschichten einen androzentrischen Bias. Porträtiert werden schwule und Trans-Männer. Weibliche Homosexualität und Trans-Frauen scheinen auch in liberalen Medien nicht ernst genommen zu werden (vgl. Amberg 2011).

\section{RESÜMEE: UMKÄMPFTES GeSCHLECHTERWISSEN VERSUS AKZEPTANZ GESCHLECHTLICHER VIELFALT IN DER PRAXIS}

In der vorliegenden exemplarischen Untersuchung konnten medienübergreifend keine nennenswerten Thematisierungskonjunkturen von ,Genderismus'-Diskursen identifiziert werden. Zugleich ist in der Frame-Analyse kombiniert mit den Analysen der Leser_innen-Kommentare eine widersprüchliche Gleichzeitigkeit der Abwehr von Verunsicherungen in medialen Angriffen auf Gender Studies und genderbewussten Sprachgebrauch einerseits und einer Akzeptanz der Liberalisierung von Geschlechterordnungen bis in konservative Medien und ihre Leser_innenschaft hinein andererseits erkennbar. Diskutiert werden kann das als medialer Ausdruck einer gesellschaftlich umkämpften Normalität oder Normalisierung der Geschlechterordnungen, die Lenz (2013) als Gleichzeitigkeit von Wandel und Persistenz im Übergang zur „flexibilisierten Geschlechterordnung“ bezeichnet. In der Abwehr von geschlechtlicher Vielfalt und einer Perspektive auf Geschlecht als sozial und kulturell konstruiert wird zum einen die vermeintliche Sicherheit der ,Normalität' einer heteronormativen Ordnung und ebensolcher Paarbeziehungen verteidigt, in der andere geschlechtliche Identitäten und Lebensweisen allenfalls als geduldete Minderheit Platz haben. Dies würde die heftigen und polemischen Angriffe auf die wissen- 
schaftliche Befassung mit Konstruktionen von Geschlecht sowie die sprachliche Sichtbarmachung von geschlechtlicher Vielfalt plausibilisieren.

Zum anderen werden - auch in den Medien - geschlechtliche Identitäten und Lebensentwürfe, die von der heteronormativen Ordnung abweichen, zunehmend als gesellschaftliche Realität akzeptiert. Beispiele dafür sind ein zunächst heterosexuelles Paar, bei dem die Frau ihr Geschlecht zum Mann angleichen ließ, als Vorbild für normativ wünschenswerte Beziehungen in der FAZ (20.06.2009), Leser_innenbriefe in der Welt, die queere Lebensweisen als normal bezeichnen, und ein Artikel in der SZ über die Proteste gegen den reformierten Bildungsplan in Baden-Württemberg, nach dem Kinder erfahren sollten, dass es neben Heterosexualität auch noch anderes gibt (SZ 04.02.2014). Diese Widersprüchlichkeit könnte darauf hinweisen, dass veränderte Praktiken akzeptabel sind, während ein Wandel der zugrundeliegenden gesellschaftlichen Ordnung weiterhin hoch umstritten ist. Fraglich bleibt bei dieser Überlegung jedoch, ob die Liberalisierung lediglich auf eine „Normalität auf Bewährung“ hinausläuft, die Heilmann (2011) am Beispiel des Outings schwuler Spitzenpolitiker beschrieben hat. In diesem Fall würden nur solche Lebensweisen, etwa die Ehe für alle, akzeptiert, die heterosexuellen Normalitätsvorstellungen entsprechen. Heteronormativität würde damit gleichsam um nicht-heterosexuelle Lebensweisen erweitert.

Ein medialer ,Genderismus'-Diskurs wird hauptsächlich auf den Feldern Gen-

der Studies und genderbewusste Sprache ausgetragen, während geschlechtliche Vielfalt als Praxis und entsprechende pädagogische Konzepte weniger umstritten zu sein scheinen. Die Resouveränisierungsdiskurse um eine wehrhafte Männlichkeit wiederum könnten eher als antifeministische denn als anti-, genderistische“ Diskurse eingeordnet werden. Hier wird nicht in erster Linie geschlechtliche Vielfalt angegriffen. Vielmehr sind zentrale Argumentationsmuster die vermeintliche Verantwortung der Frauen für die Misere der Männer, die Anrufung hegemonial männlicher Werte und damit die Aktualisierung heteronormativer Geschlechterhierarchien.

\section{LITERATUR}

Aigner, Isolde (2012): „,Schrumpfmänner“ im ,lila Imperium‘. Antifeministische Denkmuster im medialen Diskurs“, in: Andreas Kemper (Hg.), Die Maskulisten: Organisierter Antifeminismus im deutschsprachigen Raum, Münster: Unrast-Verlag, S. 46-57.

Aigner, Isolde (2013): „Antifeminismus Reloaded. Fließende Übergänge von der extremen Rechten zur bürgerlichen ,Mitte““, in: Sabine Berghahn/Ulrike Schulz (Hg.), Rechtshandbuch für Frauen- und Gleichstellungsbeauftragte, Hamburg: Dashöfer, 9/8.1 S. 1-5. 
Amberg, Elke (2011): Schön! Stark! Frei! Wie Lesben in der Presse (nicht) dargestellt werden, Sulzbach/Taunus: Ulrike Helmer.

Beck, Dorothee (2016): Politikerinnen und ihr Griff zur Macht. Mediale Repräsentationen von SPD-Spitzenkandidatinnen bei Landtagswahlen, Bielefeld: transcript.

Berbuir, Nicole/Lewandowsky, Marcel/Siri, Jasmin (2015): „The AfD and its Sympathisers: Finally a Right-Wing Populist Movement in Germany?", in: German Politics 24 (2), S. 154-178.

Bergold-Caldwell, Denise/Grubner, Barbara (2020): „Effekte der diskursiven Verknüpfung von Antifeminismus und Rassismus. Eine Fallstudie zu Orientierungskursen für neu Zugewanderte“, in: Henninger/Birsl, Antifeminismen. ,Krisen'-Diskurse mit gesellschaftsspaltendem Potential?

Billmann, Lucie (Hg.) (2015): Unheilige Allianz. Das Geflecht von christlichen Fundamentalisten und politisch Rechten am Beispiel des Widerstandes gegen den Bildungsplan in Baden-Württemberg, Berlin: Rosa-Luxemburg-Stiftung.

Blum, Roger (2011): „Leidende Leuchttürme. Über die Unentbehrlichkeit von Qualitätsmedien“, in: Blum/Bonfadelli/Imhof/Jarren, Krise der Leuchttürme öffentlicher Kommunikation. Vergangenheit und Zukunft der Qualitätsmedien, S. 714.

Blum, Roger/Bonfadelli, Heinz/Imhof, Kurt/Jarren, Otfried (Hg.) (2011): Krise der Leuchttürme öffentlicher Kommunikation. Vergangenheit und Zukunft der Qualitätsmedien, Wiesbaden: VS.

Claus, Robert (2014): Maskulismus. Antifeminismus zwischen vermeintlicher Salonfähigkeit und unverhohlenem Frauenhass, Berlin: Friedrich-Ebert-Stiftung. [http://library.fes.de/pdf-files/dialog/10861.pdf; abgerufen am 19.12.2019].

Daphi, Priska/Kocyba, Piottr/Neuber, Michael/Roose, Jochen/Rucht, Dieter/Scholl, Franziska/Sommer, Moritz/Stuppert, Wolfgang/Zajak, Sabrina (2015): Protestforschung am Limit. Eine soziologische Annäherung an PEGIDA, Frankfurt a.M.: Otto-Brenner-Stiftung. [https://www.otto-brenner-stiftung.de/fileadmin/ user_data/stiftung/02_Wissenschaftsportal/03_Publikationen/2015_Protestfor schung.pdf; abgerufen am 19.12.2019].

Dietze, Gabriele (2016): „Das ,Ereignis Köln““, in: femina politica 25 (1), S. 93102.

Entman, Robert (1993): „Framing: Toward Clarification of a Fractured Paradigm“, in: Journal of Communication 43 (4), S. 51-58.

Forster, Edgar (2006): „Männliche Resouveränisierungen“, in: feministische studien 2, S. 193-207.

Frey, Regina (2014): „Von Mythen und Vermischungen. Zur Konstruktion des ,Genderismus““, in: Regina Frey/Marc Gärtner/Manfred Köhnen/Sebastian Scheele (Hg.), Gender, Wissenschaftlichkeit und Ideologie: Argumente im Streit um Geschlechterverhältnisse, Berlin: Heinrich-Böll-Stiftung, S. 28-39. [www.gwi-boell.de/de/2013/11/20/gender-wissenschaftlichkeit-und-ideologie- 
argumente-im-streit-um-geschlechterverh\%C3\%A4ltnisse; abgerufen am 19.12. 2019].

Fritzsche, Christopher/Lang, Juliane (2020): „Die GegnerInnenschaft zur Ehe für alle: ein autoritär-regressives politisches Projekt?“, in: Henninger/Birsl, Antifeminismen. ,Krisen“-Diskurse mit gesellschaftsspaltendem Potential?

Gesterkamp, Thomas (2010): Geschlechterkampf von rechts. Wie Männerrechtler und Familienfundamentalisten sich gegen das Feindbild Feminismus radikalisieren, Bonn: Friedrich-Ebert-Stiftung. [http://library.fes.de/pdf-files/wiso/070 54.pdf; abgerufen am 19.12.2019].

GEW (Hg.) (2016): Für eine Pädagogik der Vielfalt. Argumente gegen ultrakonservative, neu-rechte und christlich-fundamentalistische Behauptungen, Frankfurt a.M.: Gewerkschaft Erziehung und Wissenschaft, Hauptvorstand.

Goffman, Erving (1994): Interaktion und Geschlecht, Frankfurt a.M.: Campus.

Hark, Sabine/Villa, Paula-Irene (Hg.) (2015): Anti-Genderismus: Sexualität und Geschlecht als Schauplätze aktueller politischer Auseinandersetzungen, Bielefeld: transcript.

Heilmann, Andreas (2011): Normalität auf Bewährung: Outings in der Politik und die Konstruktion homosexueller Männlichkeit, Bielefeld: transcript.

Henninger, Annette/Birsl, Ursula (Hg.) (2020): Antifeminismen. ,Krisen“-Diskurse mit gesellschaftsspaltendem Potential?, Bielefeld: transcipt.

Jarren, Otfried/Vogel, Ulrike (2011): „,Leitmedien“ als Qualitätsmedien. Theoretisches Konzept und Indikatoren“, in: Blum/Bonfadelli/Imhof/Jarren, Krise der Leuchttürme öffentlicher Kommunikation. Vergangenheit und Zukunft der Qualitätsmedien, S. 17-29.

Kemper, Andreas (2014a): Keimzelle der Nation? Familien- und geschlechterpolitische Positionen der AfD - eine Expertise, Berlin. [http://library.fes.de/pdffiles/dialog/10641-20140414.pdf; abgerufen am 16.03.2020].

Kemper, Andreas (2014b): Keimzelle der Nation? Teil 2. Wie sich in Europa Parteien und Bewegungen für konservative Familienwerte, gegen Toleranz und Vielfalt und gegen eine progressive Geschlechterpolitik radikalisieren, Berlin. [http://library.fes.de/pdf-files/dialog/11163.pdf; abgerufen am 19.12.2019].

Klaus, Elisabeth (2008): „Anti-Feminismus und konservativer Feminismus - eine Intervention“, in: feministische studien 26 (2), S. 176-186.

Kováts, Eszter/Põim, Maari (Hg.) (2015): Gender as Symbolic Glue. The Position and Role of Conservative and Far Right Parties in the Anti-Gender Mobilizations in Europe, Budapest: Foundation for European Progressive Studies/Friedrich-Ebert-Stiftung. [http://library.fes.de/pdf-files/bueros/budapest/113 82.pdf; abgerufen am 19.12.2019].

Krüger-Kirn, Helga/Lather, Dietger/Näser-Lather, Marion/Schumacher, Nina (2019): „Strategien zur Manipulation der Öffentlichkeit. Unwissenschaftliche Kritik an ,Gender" in Ulrich Kutscheras Gender-Paradoxon“, in: Näser-Lather/ 
Oldemeier/Beck, Backlash?! Antifeminismus in Wissenschaft, Politik und Gesellschaft, S. 128-148.

Krüger-Kirn, Helga/Tichy, Leila Zoë (2020): „Elternschaft und Gender Trouble. Inszenierungen moderner und tradierter Mutterbilder", in: Henninger/Birsl, Antifeminismen. ,Krisen“-Diskurse mit gesellschaftsspaltendem Potential?

Kuhar, Roman/Paternotte, David (2017): Anti-Gender Campaigns in Europe: Mobilizing Against Equality, London/New York: Rowman \& Littlefield International.

Lang, Juliane (2015): „Familie und Vaterland in der Krise. Der extrem rechte Diskurs um Gender", in: Hark/Villa, Anti-Genderismus: Sexualität und Geschlecht als Schauplätze aktueller politischer Auseinandersetzungen, S. 167-181.

Lang, Juliane/Peters, Ulrich (2018): Antifeminismus in Bewegung, Hamburg: Marta Press UG.

Lenz, Ilse (2011): „Der neue Antifeminismus. Der Fall Kachelmann und das Bild vom männlichen Opfer“, in: Blätter für deutsche und internationale Politik 7, S. 51-59.

Lenz, Ilse (2013): „Geschlechterkonflikte um die Geschlechterordnung im Übergang: zum neuen Antifeminismus“, in: Erna Appelt/Brigitte Aulenbacher/ Angelika Wetterer (Hg.), Gesellschaft: feministische Krisendiagnose, Münster: Westfälisches Dampfboot, S. 204-226.

Matthes, Jörg (2014): Framing, Baden-Baden: Nomos.

Mayring, Philipp (2010): Qualitative Inhaltsanalyse. Grundlagen und Techniken. 11., aktualisierte und überarbeitete Aufl., Weinheim: Beltz.

Meyen, Michael/Löblich, Maria/Pfaff-Rüdiger, Senta/Riesmeyer, Claudia (2011): Qualitative Methoden in der Kommunikationswissenschaft, Wiesbaden: VS.

Näser-Lather, Marion (2019): „,Wider den Genderismus!‘ Kritik und Polemiken gegen die Gender Studies in akademischen Kontexten“, in: Näser-Lather/Oldemeier/Beck, Backlash?! Antifeminismus in Wissenschaft, Politik und Gesellschaft, S. 105-127.

Näser-Lather, Marion (2020): „Wissenschaftler_innen vs. Gender Studies. Argumentationen, Wirkungen und Kontexte einer, wissenschafts'-politischen Debatte“, in: Henninger/Birsl, Antifeminismen. ,Krisen“-Diskurse mit gesellschaftsspaltendem Potential?

Näser-Lather, Marion/Oldemeier, Anna Lena/Beck, Dorothee (Hg.) (2019): Backlash?! Antifeminismus in Wissenschaft, Politik und Gesellschaft, Roßdorf: Ulrike Helmer.

Netzwerk Recherche (2006): Spiegel Online ist die neue Stimme im Kanon der deutschen Leitmedien. [https://netzwerkrecherche.org/blog/spiegel-online-istdie-neue-stimme-im-kanon-der-deutschen-leitmedien/; abgerufen am 19.12. 2019].

Oldemeier, Anna Lena/Backöfer, Ferdinand/Maurer, Susanne/Aleksin, Katharina (2020): „Divergenz, Ambivalenz, Kongruenz. Verhältnisbestimmungen zwi- 
schen antifeministischem Diskurs und pädagogischem Feld“, in: Henninger/ Birsl, Antifeminismen. ,Krisen'-Diskurse mit gesellschaftsspaltendem Potential?

Rieske, Thomas Viola (2011): Bildung von Geschlecht. Zur Diskussion um Jungenbenachteiligung und Feminisierung in deutschen Bildungsinstitutionen, Frankfurt a.M.: Max-Träger-Stiftung.

Rosenbrock, Hinrich (2012): Die anti-feministische Männerrechtsbewegung: Denkweisen, Netzwerke und Online-Mobilisierung, Berlin: Heinrich-BöllStiftung. [https://www.boell.de/sites/default/files/antifeministische_maenner rechtsbewegung.pdf; abgerufen am 19.12.2019].

Scheele, Sebastian (2016): Von Antifeminismus zu ,Anti-Genderismus"? Eine diskursive Verschiebung und ihre Hintergründe, Gunda-Werner-Institut in der Heinrich-Böll-Stiftung, Berlin, 31. Mai 2016.

Schmincke, Imke (2015): „Das Kind als Chiffre politischer Auseinandersetzungen am Beispiel neuer konservativer Protestbewegungen in Frankreich und Deutschland“, in: Hark/Villa, Anti-Genderismus: Sexualität und Geschlecht als Schauplätze aktueller politischer Auseinandersetzungen, S. 93-108.

Schutzbach, Frankziska/Lanwer, Michelle (2017): „,Ich kann euch alle haben. Maskulinitätsideologien und Rechtsnationalismus“, in: geschichtedergegenwart.ch. [http://geschichtedergegenwart.ch/ich-kann-euch-alle-haben/; abgerufen am 19.12.2019].

Spierings, Niels (2018): „Popular Opposition to Economic Gender Equality and Homosexual Lifestyles“, in: Mieke Verloo (Hg.), Varieties of Opposition to Gender Equality in Europe, New York: Routledge, Taylor \& Francis Group, S. 172-194.

Vorländer, Hans/Schäller, Steven/Herold, Maik (2016): Pegida: Entwicklung, Zusammensetzung und Deutung einer Empörungsbewegung, Wiesbaden: Springer VS.

West, Candace/Zimmerman, Don H. (1987): „Doing Gender“, in: Gender \& Society 1 (2), S. 125-151.

Wimbauer, Christine/Mokatef, Mona/Teschlade, Julia (2015): „Prekäre Selbstverständlichkeiten. Neun prekarisierungstheoretische Thesen zu Diskursen gegen Gleichstellungspolitik und Geschlechterforschung“, in: Hark/Villa, Anti-Genderismus: Sexualität und Geschlecht als Schauplätze aktueller politischer Auseinandersetzungen, S. 41-57.

Zastrow, Volker/Feuchtenberger, Anke (2006): Gender - Politische Geschlechtsumwandlung, Waltrop: Manuscriptum.

Ziegele (2016): Nutzerkommentare als Anschlusskommunikation. Theorie und qualitative Analyse des Diskussionswerts von online-Nachrichten, Wiesbaden: Springer VS. 


\section{TeXtKorpus Medienanalyse}

\section{Frankfurter Allgemeine Zeitung}

20.06.2009: Ein Paar

20.07.2011: Kinder-Mainstreaming macht Schule

26.07.2011: Wie lange dürfen unsere Kinder noch Kinder sein?

27.07.2011: Wer fragt eigentlich die Kinder?

28.07.2011: Welche Last wird Kindern noch aufgebürdet?

30.07.2011: Die Hintertür-Methoden der Gender-Ideologen

25.01.2012: Tumult im Hause Planck

10.07.2013: Wir fahren jetzt 14 Nächte in den Sprachurlaub

25.07.2013: Feministische Linguistik

03.08.2013: Die Mensch/innen/heit

23.10.2014: Aufklärung oder Anleitung zum Sex

27.10.2014: Irrsinn; Selbsternannte Aufklärer unserer Kinder; Missbrauch, Umerziehung der Gesellschaft; Warnender Tenor

30.10.2014: Unwissenheit hat noch niemandem geholfen; Es tobt ein Kulturkampf um unsere Kinder; Nieder mit der Gender-Ideologie

31.12.2014: Sollen sich die Kinder doch wehren

12.04.2014: Warum Frauen am DVD-Spieler scheitern

21.05.2015: Heldenhafte Spermien und wachgeküsste Eizellen

06.06.2015: Meinungsfreiheit

27.06.2015: Wen stört es wirklich?

16.10.2015: Das vernachlässigte Geschlecht

18.08.2016: Burka und Niquab gehören nicht zu Deutschland

23.08.2016: Die Grenze der Toleranz

05.11.2016: ProfessX trifft Studierende

12.11.2016: Keine Sternchen fürs Sternchen (Mitgemeint; Rechtlich äußerst bedenklich; Überflüssiger Firlefanz; Nicht einmal dumpf erahnen; Gerechtigkeitsfanatikern ausgeliefert; Zukunft findet ohne uns statt; Liebe Mitbürgerx; Machen, nicht labern; Ich stolpere über Schrägstriche; Auf Comic-Niveau; Schutz vor Gender-Gaga)

22.06.2017: Dem Beispiel der Pinguine und Flamingos folgen (zu FAZ: 30.05.2015)

\section{Spiegel online}

08.05.2007: Als Vati über Mutti rollte

19.10.2007: Adam und Steve im Paradies

15.04.2010: Abschied vom Macho-Mann

17.04.2010: Brecht als Comic 
16.01.2013: Backpfeife für Studierende

04.07.2013: Die Präsidentin/der Präsident muss gehen

04.09.2015: Jung, attraktiv, muss gut kochen können

12.09.2015: Die gekränkten Kritiker

21.09.2015: Verstecken bis zur Verzweiflung

13.12.2015: Homosexuell im Alpental

30.05.2016: Aua, Papa

10.11.2016: Ist der Ruf erst ruiniert

Die Leser_innen-Kommentare haben das gleiche Datum wie die jeweiligen Artikel; zur Orientierung sind im Text die jeweiligen User-Namen angegeben.

\section{Süddeutsche Zeitung}

25.06.2010: Gleichheit ist gar nicht das Ziel

11.03.2011: Kerle in Angst

10.09.2013: Streiflicht

17.09.2013: Das Knacken von Cyborg-Gelenken

25.09.2013: Die deutsche Teilung

14.10.2013: Streiflicht

04.02.2014: Kulturkampf in Südwest

02.05.2014: Streiflicht

10.01.2015: Mr. Unisex

03.03.2015: Wie Fische ohne Fahrrad

11.02.2016: Legal, illegal, alles egal

16.04.2016: Krampfzone

21.04.2016: Forschungsgeld, das bestens aufgehoben ist (Ist das Ihr Ernst? In den kulturellen Kontext setzen; Zu persönlich, zu krass; Basisliteratur vieler Fächer; Soziale Konstrukte; Ins Herz; Unschätzbarer Beitrag; Sozial-, nicht Naturwissenschaft)

27.08.2016: Kerlschmelze

\section{Die Welt}

26.10.2007: Kinderliebe, Elternliebe

29.10.2007: Verstöße gegen Elternrecht; Zu viel Sex überall; Sofia Kuby; Micha Heimsoth

24.04.2008: Die neue Schule der Jungen

03.06.2013: Das Leben ist gar nicht so rosa

14.06.2013: Grenzenlos emanzipiert

17.06.2013: Mehr Gelassenheit; Keine Hybris

03.05.2014: Professx statt Professor? So irre ist das nicht

06.05.2014: Gendergewirr 
28.06.2014: Faustschlag mit lackierten Nägeln

19.11.2014: GenderInnen-Wahn

23.06.2015: Schwaben legen sich quer gegen queer

26.06.2015: Die Stunde der Sex-Ideologen

04.07.2015: Kinderseelen

28.12.2015: Deutsch und deutscher

30.12.2015: Lautstarke Lobby

11.02.2016: Ballt das Händchen doch mal zur Faust

23.02.2016: Neue Väter, neue Männer

12.04.2016: Der Justizpuritaner

13.04.2016: Abschaffen

23.05.2016: Sysiphos war ein glücklicher Mensch

\section{Weitere QuelLen}

Frankfurter Allgemeine Zeitung

24.05.2006: Löwe, Panther, Pilz

19.06.2006: Politische Geschlechtsumwandlung

07.09.2006: Der kleine Unterschied

28.02.2014: Kretschmann für Gender-Theorie

05.10.2016: Seid fruchtbar und mehret euch

Junge Freiheit 07.07.2006: Neue Spielwiese für Feministinnen

rbb24 (2019): Kramp-Karrenbauer erntet Shitstorm nach Intersexuellen-Spott. rbb24. [https://www.rbb24.de/politik/beitrag/2019/03/Kramp-Karrenbauer-Shit storm-beleidigt-Intersexuelle.html; abgerufen am 19.12.2019].

Der Spiegel 01/2007: Der neue Mensch

Spiegel online 03.08.2016: Papst kritisiert Gender-Lehre an Schulen

Verein deutsche Sprache (2019): Schluss mit dem Gender-Unfug. 06.03.2019.

[https://vds-ev.de/gegenwartsdeutsch/gendersprache/gendersprache-unterschrif ten/schluss-mit-dem-gender-unfug/; abgerufen am 19.12.2019].

Die Welt 27.01.2016: AfD ist die Partei der älteren Männer

Zastrow, Volker/Feuchtenberger, Anke (2006): Gender - Politische Geschlechtsumwandlung, Waltrop: Manuscriptum. 